\title{
Christoffel Symbols and Chiral Properties of the Space-Time Geometry for the Atomic Electron States
}

\author{
Claude Daviau, Jacques Bertrand \\ Fondation Louis de Broglie, Paris, France \\ Email: daviau.claude@orange.fr, bertrandjacques-m@orange.fr
}

\begin{abstract}
How to cite this paper: Daviau, C. and Bertrand, J. (2021) Christoffel Symbols and Chiral Properties of the Space-Time Geometry for the Atomic Electron States. Journal of Modern Physics, 12, 483-512.

https://doi.org/10.4236/jmp.2021.124033
\end{abstract}

Received: February 21, 2021

Accepted: March 15, 2021

Published: March 18, 2021

Copyright $\odot 2021$ by author(s) and Scientific Research Publishing Inc. This work is licensed under the Creative Commons Attribution International License (CC BY 4.0).

http://creativecommons.org/licenses/by/4.0/

\begin{abstract}
Quantum electron states, in the case of an improved Dirac equation, are linked to the Christoffel symbols of the connection of space-time geometry. Each solution of the wave equation, in the case of the hydrogen atom induces a connection which is completely calculated. This allows us to discover the global and chiral properties of the space-time connection, with spin 2 .
\end{abstract}

\section{Keywords}

Quantum Mechanics, Clifford Algebra, Differential Geometry, Dirac Equation, Hydrogen Atom, Chirality

\section{Introduction}

After L. de Broglie's discovery of the quantum wave [1], Dirac formulated his relativistic wave equation for the electron [2], correcting the non-relativistic Schrödinger equation, and conserving the probability density linked to the wave. The main success of this wave equation was its application to the case of the hydrogen atom: all the expected quantum numbers were obtained, as well as the true number of states, and the true energy levels [3]. Moreover, the Dirac equation explained the spin $1 / 2$ of the electron. The main problem was the presence of negative energies which were then accounted for as due to charge conjugation.

Following de Broglie's ideas on the necessity of non-linearity to unify quantum physics and gravitation, an improved Dirac equation was studied [4]-[43]. This relativistic wave equation was extended to a wave equation of all fermions and anti-fermions of the first generation, as described in "Developing a Theory of Everything" [41]. The resolution of the wave equation in the case of the hy- 
drogen atom was completed in [42]. The aim of the present work is the use of these solutions as physical examples of the differential geometry linked to the quantum wave. The calculation of the Christoffel symbols was previously made only in the case of plane waves: geometry of space-time was characterized by a not null torsion and a null curvature. The torsion was linked to the mass term of the electron. The case of the electron in the hydrogen atom is much more complicated indeed, and also much more interesting because it will allow us to encounter the geometric aspect of chirality in quantum mechanics.

\section{Tensorial Densities without Derivative}

Early on, the Dirac theory encountered 16 of the 36 tensorial densities that may be computed without derivatives from the electron wave. This is easy to see with the Pauli algebra [5] [7] [8]. From the wave of the electron, $\phi=\phi(\mathrm{x})$, we get four space-time vectors (16 densities):

$$
D_{\mu}:=\phi \sigma_{\mu} \phi^{\dagger}, \mu=0,1,2,3 ; \phi:=\sqrt{2}\left(\begin{array}{cc}
\xi_{1} & -\bar{\eta}_{2} \\
\xi_{2} & \bar{\eta}_{1}
\end{array}\right) ; \phi^{\dagger}=\sqrt{2}\left(\begin{array}{cc}
\bar{\xi}_{1} & \bar{\xi}_{2} \\
-\eta_{2} & \eta_{1}
\end{array}\right) .
$$

And we get 20 densities (2 with $S_{0}$ and 6 for each $S_{k}$ ) as the components of:

$$
S_{\mu}:=\phi \sigma_{\mu} \bar{\phi} ; \bar{\phi}:=\sqrt{2}\left(\begin{array}{cc}
\bar{\eta}_{1} & \bar{\eta}_{2} \\
-\xi_{2} & \xi_{1}
\end{array}\right) .
$$

The previous equalities use the three well-known Pauli matrices $\sigma_{1}, \sigma_{2}, \sigma_{3}$ and we let $\sigma_{0}:=1$, identifying real numbers and scalar matrices. The 16 densities of the early theory were the components of the probability current $\mathrm{J}:=D_{0}$, the $\mathrm{K}:=D_{3}$ current, the $S_{3}$ bivector (6 components) and the $\Omega_{1}$ and $\Omega_{2}$ invariants, satisfying:

$$
S_{0}=\phi \bar{\phi}=\operatorname{det}(\phi)=2\left(\xi_{1} \bar{\eta}_{1}+\xi_{2} \bar{\eta}_{2}\right)=\rho \mathrm{e}^{i \beta}=\Omega_{1}+i \Omega_{2}=2\left(\eta_{1} \eta_{2}\right)^{\dagger}\left(\begin{array}{l}
\xi_{1} \\
\xi_{2}
\end{array}\right),
$$

where $\beta$ is the Yvon-Takabayasi angle. We just encountered the right $(\xi)$ and left $(\eta)$ parts of the wave. We also need:

$$
\begin{gathered}
\hat{\phi}:=\bar{\phi}^{\dagger}=\sqrt{2}\left(\begin{array}{cc}
\eta_{1} & -\bar{\xi}_{2} \\
\eta_{2} & \bar{\xi}_{1}
\end{array}\right) ; \mathrm{R}:=\sqrt{2}\left(\begin{array}{ll}
\xi_{1} & 0 \\
\xi_{2} & 0
\end{array}\right) ; \hat{\mathrm{L}}:=\sqrt{2}\left(\begin{array}{cc}
\eta_{1} & 0 \\
\eta_{2} & 0
\end{array}\right) \\
D_{R}:=\mathrm{RR}^{\dagger} ; D_{L}:=\mathrm{LL}^{\dagger} .
\end{gathered}
$$

Currents $D_{R}$ and $D_{L}$ are the chiral currents that dominate in weak interactions. They satisfy:

$$
D_{0}=D_{R}+D_{L} ; D_{3}=D_{R}-D_{L} .
$$

From the beginning of Dirac's research, relativistic quantum mechanics is a non-local theory, because the wave equation is not the only condition that governs the dynamics of the wave:

$$
\iiint \mathrm{d} v \frac{\mathrm{J}^{0}}{\hbar c}=1 .
$$


We explained in 1.5 .5 of [41] how this condition is a physical law that issues from the principle of equivalence. This law, joined with the invariance under the greater group $\mathrm{Cl}_{3}^{*}$, implies the quantization of the kinetic momentum [41] [43]. The four $D_{\mu}$ currents form an orthogonal basis of space-time because they satisfy:

$$
D_{\mu} \cdot D_{v}=0, \mu \neq v ; D_{0} \cdot D_{0}=\rho^{2}=-D_{1} \cdot D_{1}=-D_{2} \cdot D_{2}=-D_{3} \cdot D_{3} .
$$

The $\phi$ wave of the electron defines a similitude:

$$
\begin{gathered}
D_{\mathrm{x}}: X=X^{\mu} \sigma_{\mu} \mapsto \mathrm{x}=\phi X \phi^{\dagger}=\phi X^{v} \sigma_{v} \phi^{\dagger}=X^{v} D_{v}, \\
D_{v}=D_{v}^{\mu} \sigma_{\mu}=\phi \sigma_{v} \phi^{\dagger} .
\end{gathered}
$$

This variable basis is then associated with an affine connection. This also allows us to use the Cartan's mobile basis $\left(D_{0}, D_{1}, D_{2}, D_{3}\right)$. This connection was first studied in [15]. We let:

$$
\begin{gathered}
\partial_{v}=\frac{\partial}{\partial X^{v}}=D_{v}^{\mu} \partial_{\mu} ; \mathrm{dx}=\mathrm{d} X^{v} D_{v}, \\
\mathrm{~d} D_{\mu}=\Gamma_{\mu \nu}^{\beta} \mathrm{d} X^{v} D_{\beta} .
\end{gathered}
$$

This gives if $\rho \neq 0$ :

$$
\begin{gathered}
\mathrm{dx}=\mathrm{dx}^{\mu} \sigma_{\mu}=D_{v}^{\mu} \sigma_{\mu} \mathrm{d} X^{v}=D_{v} \mathrm{~d} X^{v}, \\
D_{v}=\phi \sigma_{v} \phi^{\dagger}=D_{v}^{\mu} \sigma_{\mu} ; \sigma_{\mu}=\left(D^{-1}\right)_{\mu}^{\beta} D_{\beta} .
\end{gathered}
$$

Now we use the similitude $\bar{D}$ such as:

$$
\bar{D}(\mathrm{x})=\bar{\phi} \mathrm{x} \hat{\phi} .
$$

We have

$$
\begin{aligned}
& D \circ \bar{D}(\mathrm{x})=D[\bar{D}(\mathrm{x})]=\phi \bar{\phi} \times \hat{\phi} \phi^{\dagger}=\rho \mathrm{e}^{i \beta} \mathrm{x} \rho \mathrm{e}^{-i \beta}=\rho^{2} \mathrm{x}, \\
& D \circ\left(\rho^{-2} \bar{D}\right)(\mathrm{x})=\mathrm{x}, \\
& D^{-1}(\mathrm{x})=\rho^{-2} \bar{D}(\mathrm{x}) .
\end{aligned}
$$

And we get:

$$
\begin{aligned}
\mathrm{d} D_{\mu} & =\partial_{\nu}\left(D_{\mu}\right) \mathrm{d} X^{v}=\partial_{\nu}\left(D_{\mu}^{\xi} \sigma_{\xi}\right) \mathrm{d} X^{v}=\partial_{\nu}\left(D_{\mu}^{\xi}\right) \sigma_{\xi} \mathrm{d} X^{v} \\
& =\partial_{v}\left(D_{\mu}^{\xi}\right)\left(D^{-1}\right)_{\xi}^{\beta} D_{\beta} \mathrm{d} X^{v}=\Gamma_{\mu \nu}^{\beta} D_{\beta} \mathrm{d} X^{v} .
\end{aligned}
$$

Therefore the coefficients of the connection satisfy:

$$
\Gamma_{\mu v}^{\beta}=\partial_{v}\left(D_{\mu}^{\xi}\right)\left(D^{-1}\right)_{\xi}^{\beta} ; \partial_{v}=D_{v}^{\tau} \partial_{\tau} .
$$

By using the $\bar{D}$ similitude we get:

$$
\Gamma_{\mu v}^{\beta}=\rho^{-2} \partial_{v}\left(D_{\mu}^{\xi}\right) \bar{D}_{\xi}^{\beta} ; \partial_{v}=D_{v}^{\tau} \partial_{\tau} .
$$

Since $\bar{D}_{0}^{0}=D_{0}^{0}$ and $\bar{D}_{j}^{0}=-D_{0}^{j}$ we have:

$$
\Gamma_{0 v}^{0}=\Gamma_{1 v}^{1}=\Gamma_{2 v}^{2}=\Gamma_{3 v}^{3}=\partial_{v}[\ln (\rho)]=D_{v}^{\mu} \partial_{\mu}[\ln (\rho)] .
$$


The Christoffel symbols of this connection satisfy (see [41] 4.1):

$$
\mathrm{x}^{\prime \alpha}=\mathrm{x}^{\alpha}+\mathrm{dx}^{\alpha}+\Gamma_{\beta \gamma}^{\alpha} \mathrm{x}^{\beta} \mathrm{dx} \mathrm{x}^{\gamma},
$$

For the complete calculation of these coefficients of the connection we need the following quantities:

$$
\mathcal{S}_{(k)}+i \mathcal{S}_{(k)}^{\prime}:=\frac{\nabla S_{k}^{\dagger}}{\operatorname{det}\left(\phi^{\dagger}\right)} ; \mathcal{A}_{(k)}+i \mathcal{A}_{(k)}^{\prime}:=\frac{A S_{k}^{\dagger}}{\operatorname{det}\left(\phi^{\dagger}\right)} .
$$

With the improved wave equation of the electron, we obtained in D.4 of [41] the following symbols:

$$
\begin{gathered}
\Gamma_{1 \mu}^{0}=D_{\mu} \cdot\left[\mathcal{S}_{(1)}-2 q \mathcal{A}_{(2)}\right]+2 m \rho \delta_{\mu}^{2}, \\
\Gamma_{2 \mu}^{0}=D_{\mu} \cdot\left[\mathcal{S}_{(2)}+2 q \mathcal{A}_{(1)}\right]-2 m \rho \delta_{\mu}^{1}, \\
\Gamma_{3 \mu}^{0}=D_{\mu} \cdot \mathcal{S}_{(3)}, \\
\Gamma_{3 \mu}^{2}=-D_{\mu} \cdot\left[\mathcal{S}_{(1)}^{\prime}-2 q \mathcal{A}_{(2)}^{\prime}\right], \\
\Gamma_{1 \mu}^{3}=-D_{\mu} \cdot\left[\mathcal{S}_{(2)}^{\prime}+2 q \mathcal{A}_{(1)}^{\prime}\right], \\
\Gamma_{2 \mu}^{1}=-D_{\mu} \cdot\left[\mathcal{S}_{(3)}^{\prime}+2 q A\right]-2 m \rho \delta_{\mu}^{0}, \\
\Gamma_{0 \mu}^{0}=\Gamma_{1 \mu}^{1}=\Gamma_{2 \mu}^{2}=\Gamma_{3 \mu}^{3}=D_{\mu} \cdot \nabla \ln (\rho), \\
\Gamma_{0 v}^{j}=\Gamma_{j v}^{0}, j=1,2,3, \\
\Gamma_{k \nu}^{j}=-\Gamma_{j v}^{k}, \quad j=1,2,3, k=1,2,3, k \neq j .
\end{gathered}
$$

with $\delta_{0}^{0}=1, \delta_{j}^{j}=-1, j=1,2,3$ and $\delta_{\mu}^{v}=0, \mu \neq v$. Since these tensorial densities are defined through partial derivatives of the $\phi$ wave of the electron, these definitions need the wave equation that we present now.

\section{Improved Dirac Wave Equation}

The improvement of the Dirac equation was first presented in the frame of the Clifford algebra of space-time used by Hestenes, who considered the $\gamma_{\mu}$ matrices of the Dirac theory as a basis of space-time [44] [45]. Read now in $\mathrm{Cl}_{3}$ the Lagrangian density of the Dirac equation is:

$$
\mathcal{L}:=\left\langle\bar{\phi} \nabla \hat{\phi} \sigma_{21}+\bar{\phi} q A \hat{\phi}\right\rangle+m \rho \cos (\beta),
$$

where $\sigma_{21}:=\sigma_{2} \sigma_{1}=-i \sigma_{3}, A$ is the electromagnetic potential space-time vector and $\langle X\rangle$ is the real part of $X$. The improved wave equation is obtained by simplifying the Lagrangian density as:

$$
\mathcal{L}:=\left\langle\bar{\phi} \nabla \hat{\phi} \sigma_{21}+\bar{\phi} q A \hat{\phi}\right\rangle+m \rho .
$$

The improved equation which comes from this simplified Lagrangian density reads:

$$
0=\nabla \hat{\phi} \sigma_{21}+q A \hat{\phi}+m \mathrm{e}^{-i \beta} \phi
$$


This non-linear wave equation has the Dirac equation as linear approximation when the Yvon-Takabayasi $\beta$ angle is null or negligible:

$$
0=\nabla \hat{\phi} \sigma_{21}+q A \hat{\phi}+m \phi
$$

The mass term of the improved Equation (32) comes from Lochak's theory of the magnetic monopole [46]-[53] in the particular case where the Dirac Lagrangian is the linear approximation of the simplified Lagrangian density. The first improvement of this simplification is the resolution of the problem of the negative energies, because the positron is no longer associated with non-physical negative energy (see for instance [41] 1.5.3 and 1.5.6). Another improvement is the partial decoupling of the left and right spinors, the wave equation also reads:

$$
0=\nabla \hat{\phi} \sigma_{21}+(q A+m v) \hat{\phi} ; \mathrm{v}:=\frac{D_{0}}{\rho} .
$$

The momentum-energy of the electron $q A+m v$ is the sum of an electromagnetic part $q A$ and an inertial part $m v$. Hence the improved equation may be generalized to all fermions, it is compatible with the entire gauge group of the Standard Model, $U(1) \times S U(2) \times S U(3)$, and it is also compatible with the relativistic invariance of general relativity. This is the reason for the appearance of the Christoffel symbols that we will calculate from the solutions of the improved equation in the case of the hydrogen atom.

\section{Resolution in the Case of the Hydrogen Atom}

The Dirac equation was solved as early as 1928 by the mathematician C. G. Darwin using the previous resolution of the non-relativistic equation for an electron with spin found by Pauli. This method used kinetic momentum operators, which is ill-suited to the resolution of a non-linear equation like (32). Fortunately another method exists, found more recently by H. Krüger [54], who discovered a very astute method of separation of variables in spherical coordinates. This uses:

$$
x^{1}=r \sin \theta \cos \varphi ; x^{2}=r \sin \theta \sin \varphi ; x^{3}=r \cos \theta .
$$

We use the following notations:

$$
\begin{gathered}
i_{1}:=\sigma_{23}=i \sigma_{1} ; i_{2}:=\sigma_{31}=i \sigma_{2} ; i_{3}:=\sigma_{12}=i \sigma_{3}, \\
S:=\mathrm{e}^{-\frac{\varphi}{2} i_{3}} \mathrm{e}^{-\frac{\theta}{2} i_{2}} ; \Omega:=\hat{\Omega}=r^{-1}(\sin \theta)^{-\frac{1}{2}} S, \\
\vec{\partial}^{\prime}:=\sigma_{3} \partial_{r}+\frac{1}{r} \sigma_{1} \partial_{\theta}+\frac{1}{r \sin \theta} \sigma_{2} \partial_{\varphi} .
\end{gathered}
$$

H. Krüger obtained the remarkable identity:

$$
\vec{\partial}=\Omega \vec{\partial}^{\prime} \Omega^{-1},
$$

which with:

$$
\nabla^{\prime}:=\partial_{0}-\vec{\partial}^{\prime}=\partial_{0}-\left(\sigma_{3} \partial_{r}+\frac{1}{r} \sigma_{1} \partial_{\theta}+\frac{1}{r \sin \theta} \sigma_{2} \partial_{\varphi}\right)
$$

gives also: 


$$
\Omega^{-1} \nabla=\nabla^{\prime} \Omega^{-1} .
$$

For either the Dirac equation or the improved equation, to obtain the separation of the temporal variable $x^{0}=c t$ and the angular variable $\varphi$ from the radial variable $r$ and the other angular variable $\theta$, we let:

$$
\phi=\Omega X \mathrm{e}^{\zeta_{3}} ; \zeta=\lambda \varphi-E x^{0}+\delta,
$$

where $X$ is a function, with value in the Pauli algebra, of only $r$ and $\theta, \hbar c E$ is the energy of the electron, and $\delta$ is an arbitrary phase that plays no role here because the wave equations are electric gauge invariant. We get:

$$
\Omega^{-1} \phi=X \mathrm{e}^{\zeta i_{3}} ; \Omega^{-1} \hat{\phi}=\hat{X} \mathrm{e}^{\zeta i_{3}} .
$$

For the $\rho$ density that satisfies (7) we also have:

$$
\begin{gathered}
\rho \mathrm{e}^{i \beta}=\operatorname{det}(\phi)=\operatorname{det}(\Omega) \operatorname{det}(X) \operatorname{det}\left(\mathrm{e}^{\zeta i_{3}}\right), \\
\operatorname{det}(\Omega)=r^{-2}(\sin \theta)^{-1} ; \operatorname{det}\left(\mathrm{e}^{\xi_{3}}\right)=1 ; \rho \mathrm{e}^{i \beta}=\frac{\operatorname{det}(X)}{r^{2} \sin \theta} .
\end{gathered}
$$

Then if we let:

$$
\rho_{X} \mathrm{e}^{i \beta_{X}}:=\operatorname{det}(X)
$$

we get:

$$
\rho=\frac{\rho_{X}}{r^{2} \sin \theta} ; \beta=\beta_{X} .
$$

Then the Yvon-Takabayasi angle depends neither on the time nor on the $\varphi$ angle. It depends only on $r$ and $\theta$. Therefore the separation of variables can begin similarly for both the Dirac equation and the improved equation. We have:

$$
\begin{gathered}
\nabla^{\prime} \Omega^{-1} \hat{\phi}=\left(\partial_{0}-\sigma_{3} \partial_{r}-\frac{1}{r} \sigma_{1} \partial_{\theta}-\frac{1}{r \sin \theta} \sigma_{2} \partial_{\varphi}\right)\left[\hat{X} \mathrm{e}^{\zeta i_{3}}\right], \\
\partial_{0}\left(\hat{X} \mathrm{e}^{\zeta i_{3}}\right)=-E \hat{X} i_{3} \mathrm{e}^{\zeta i_{3}} ; \partial_{r}\left(\hat{X} \mathrm{e}^{\zeta i_{3}}\right)=\left(\partial_{r} \hat{X}\right) \mathrm{e}^{\zeta i_{3}} \\
\partial_{\theta}\left(\hat{X} \mathrm{e}^{\zeta i_{3}}\right)=\left(\partial_{\theta} \hat{X}\right) \mathrm{e}^{\zeta i_{3}} ; \partial_{\varphi}\left(\hat{X} \mathrm{e}^{\zeta i_{3}}\right)=\lambda \hat{X} i_{3} \mathrm{e}^{\zeta i_{3}} .
\end{gathered}
$$

We then get:

$$
\nabla \hat{\phi}=\Omega\left(-E \hat{X} i_{3}-\sigma_{3} \partial_{r} \hat{X}-\frac{1}{r} \sigma_{1} \partial_{\theta} \hat{X}-\frac{\lambda}{r \sin \theta} \sigma_{2} \hat{X} i_{3}\right) \mathrm{e}^{\zeta i_{3}} .
$$

For the hydrogen atom we have:

$$
q A=q A^{0}=-\frac{\alpha}{r} ; \alpha=\frac{e^{2}}{\hbar c},
$$

where $\alpha$ is the fine structure constant. We have:

$$
q A \hat{\phi} \sigma_{12}=-\frac{\alpha}{r} \hat{\phi} i_{3}=-\frac{\alpha}{r} \Omega \hat{X} i_{3} \mathrm{e}^{\zeta i_{3}}=\Omega\left(-\frac{\alpha}{r} \hat{X} i_{3}\right) \mathrm{e}^{\zeta i_{3}} .
$$

Also the improved Equation (32) becomes:

$$
-E \hat{X} i_{3}-\sigma_{3} \partial_{r} \hat{X}-\frac{1}{r} \sigma_{1} \partial_{\theta} \hat{X}-\frac{\lambda}{r \sin \theta} \sigma_{2} \hat{X} i_{3}-\frac{\alpha}{r} \hat{X} i_{3}+m \mathrm{e}^{-i \beta} X i_{3}=0 .
$$


This means:

$$
\left(E+\frac{\alpha}{r}\right) \hat{X} i_{3}+\sigma_{3} \partial_{r} \hat{X}+\frac{1}{r} \sigma_{1} \partial_{\theta} \hat{X}+\frac{\lambda}{r \sin \theta} \sigma_{2} \hat{X} i_{3}=m \mathrm{e}^{-i \beta} X i_{3},
$$

while the Dirac equation gives:

$$
\left(E+\frac{\alpha}{r}\right) \hat{X} i_{3}+\sigma_{3} \partial_{r} \hat{X}+\frac{1}{r} \sigma_{1} \partial_{\theta} \hat{X}+\frac{\lambda}{r \sin \theta} \sigma_{2} \hat{X} i_{3}=m X i_{3} .
$$

Now we let:

$$
X:=\left(\begin{array}{cc}
R_{1} & -\bar{L}_{2} \\
R_{2} & \bar{L}_{1}
\end{array}\right) ; \operatorname{det}(X)=R_{1} \bar{L}_{1}+R_{2} \bar{L}_{2},
$$

where $R_{1}, R_{2}, L_{1}, L_{2}$ are functions with complex values of the real variables $r$ and $\theta$. We get:

$$
\hat{X}=\left(\begin{array}{cc}
L_{1} & -\bar{R}_{2} \\
L_{2} & \bar{R}_{1}
\end{array}\right) ; X^{\dagger}=\left(\begin{array}{cc}
\bar{R}_{1} & \bar{R}_{2} \\
-L_{2} & L_{1}
\end{array}\right) ; \bar{X}=\hat{X}^{\dagger}=\left(\begin{array}{cc}
\bar{L}_{1} & \bar{L}_{2} \\
-R_{2} & R_{1}
\end{array}\right) .
$$

Therefore the improved equation reads:

$$
\begin{aligned}
& i\left(E+\frac{\alpha}{r}\right)\left(\begin{array}{cc}
L_{1} & \bar{R}_{2} \\
L_{2} & -\bar{R}_{1}
\end{array}\right)+\left(\begin{array}{cc}
\partial_{r} L_{1} & -\partial_{r} \bar{R}_{2} \\
-\partial_{r} L_{2} & -\partial_{r} \bar{R}_{1}
\end{array}\right)+\frac{1}{r}\left(\begin{array}{cc}
\partial_{\theta} L_{2} & \partial_{\theta} \bar{R}_{1} \\
\partial_{\theta} L_{1} & -\partial_{\theta} \bar{R}_{2}
\end{array}\right) \\
& +\frac{\lambda}{r \sin \theta}\left(\begin{array}{cc}
L_{2} & -\bar{R}_{1} \\
-L_{1} & -\bar{R}_{2}
\end{array}\right)=i \mathrm{e}^{-i \beta}\left(\begin{array}{cc}
m R_{1} & m \bar{L}_{2} \\
m R_{2} & -m \bar{L}_{1}
\end{array}\right) .
\end{aligned}
$$

Conjugating the equations containing the conjugates we obtain the system:

$$
\begin{aligned}
& i\left(E+\frac{\alpha}{r}\right) L_{1}+\partial_{r} L_{1}+\frac{1}{r}\left(\partial_{\theta}+\frac{\lambda}{\sin \theta}\right) L_{2}=i m \mathrm{e}^{-i \beta} R_{1}, \\
& -i\left(E+\frac{\alpha}{r}\right) R_{2}-\partial_{r} R_{2}+\frac{1}{r}\left(\partial_{\theta}-\frac{\lambda}{\sin \theta}\right) R_{1}=-i m \mathrm{e}^{i \beta} L_{2}, \\
& i\left(E+\frac{\alpha}{r}\right) L_{2}-\partial_{r} L_{2}+\frac{1}{r}\left(\partial_{\theta}-\frac{\lambda}{\sin \theta}\right) L_{1}=i m \mathrm{e}^{-i \beta} R_{2}, \\
& -i\left(E+\frac{\alpha}{r}\right) R_{1}+\partial_{r} R_{1}+\frac{1}{r}\left(\partial_{\theta}+\frac{\lambda}{\sin \theta}\right) R_{2}=-i m \mathrm{e}^{i \beta} L_{1} .
\end{aligned}
$$

Next we let:

$$
R_{1}:=A U ; L_{2}:=B V ; R_{2}:=C V ; L_{1}:=D U,
$$

where $A, B, C$ and $D$ are functions of $r$ while $U$ and $V$ are functions of $\theta$. The (60) system becomes:

$$
\begin{aligned}
& i\left(E+\frac{\alpha}{r}\right) D U+D^{\prime} U+\frac{1}{r}\left(V^{\prime}+\frac{\lambda}{\sin \theta} V\right) B=i m \mathrm{e}^{-i \beta} A U, \\
& -i\left(E+\frac{\alpha}{r}\right) C V-C^{\prime} V+\frac{1}{r}\left(U^{\prime}-\frac{\lambda}{\sin \theta} U\right) A=-i m \mathrm{e}^{i \beta} B V, \\
& i\left(E+\frac{\alpha}{r}\right) B V-B^{\prime} V+\frac{1}{r}\left(U^{\prime}-\frac{\lambda}{\sin \theta} U\right) D=i m \mathrm{e}^{-i \beta} C V, \\
& -i\left(E+\frac{\alpha}{r}\right) A U+A^{\prime} U+\frac{1}{r}\left(V^{\prime}+\frac{\lambda}{\sin \theta} V\right) C=-i m \mathrm{e}^{i \beta} D U .
\end{aligned}
$$


Then using a $\kappa$ constant satisfying:

$$
U^{\prime}-\frac{\lambda}{\sin \theta} U=-\kappa V ; V^{\prime}+\frac{\lambda}{\sin \theta} V=\kappa U,
$$

the (62) system becomes:

$$
\begin{aligned}
& i\left(E+\frac{\alpha}{r}\right) D+D^{\prime}+\frac{\kappa}{r} B=i m \mathrm{e}^{-i \beta} A \\
& -i\left(E+\frac{\alpha}{r}\right) C-C^{\prime}-\frac{\kappa}{r} A=-i m \mathrm{e}^{i \beta} B \\
& i\left(E+\frac{\alpha}{r}\right) B-B^{\prime}-\frac{\kappa}{r} D=i m \mathrm{e}^{-i \beta} C \\
& -i\left(E+\frac{\alpha}{r}\right) A+A^{\prime}+\frac{\kappa}{r} C=-i m \mathrm{e}^{i \beta} D
\end{aligned}
$$

\section{Christoffel Symbols}

We need to calculate $\nabla(\ln \rho)$ and the $\nabla S_{k}^{\dagger}, k=1,2,3$ terms. We use:

$$
\begin{gathered}
\nabla(\ln \rho)=\frac{1}{2}\left[\frac{\nabla\left(\hat{\phi} \phi^{\dagger}\right)}{\hat{\phi} \phi^{\dagger}}+\left(\frac{\nabla\left(\hat{\phi} \phi^{\dagger}\right)}{\hat{\phi} \phi^{\dagger}}\right)^{\dagger}\right] ; \mathcal{S}_{(3)}+i \mathcal{S}_{(3)}^{\prime}=\frac{\nabla\left(\hat{\phi} \sigma_{3} \phi^{\dagger}\right)}{\hat{\phi} \phi^{\dagger}}, \\
\mathcal{S}_{(3)}=\frac{1}{2}\left[\frac{\nabla\left(\hat{\phi} \sigma_{3} \phi^{\dagger}\right)}{\hat{\phi} \phi^{\dagger}}+\left(\frac{\nabla\left(\hat{\phi} \sigma_{3} \phi^{\dagger}\right)}{\hat{\phi} \phi^{\dagger}}\right)^{\dagger}\right] \\
\nabla\left(\hat{\phi} \phi^{\dagger}\right)=\Omega \nabla^{\prime}\left(\hat{X} X^{\dagger} \Omega^{\dagger}\right) ; \nabla\left(S_{3}^{\dagger}\right)=\Omega \nabla^{\prime}\left(\hat{X} \sigma_{3} X^{\dagger} \Omega^{\dagger}\right), \\
\nabla\left(S_{1}^{\dagger} \pm i S_{2}^{\dagger}\right)=\Omega \nabla^{\prime}\left[\hat{X} \mathrm{e}^{2 \zeta i_{3}}\left(\sigma_{1} \pm i \sigma_{2}\right) X^{\dagger} \Omega^{\dagger}\right] .
\end{gathered}
$$

These terms have the form:

$$
\Omega \nabla^{\prime}\left(M \Omega^{\dagger}\right)=\Omega\left(\nabla^{\prime} M\right) \Omega^{\dagger}+\Omega \underline{\nabla}^{\prime} M \underline{\Omega}^{\dagger},
$$

where the underlined refers to:

$$
\underline{\nabla}^{\prime} M \underline{\Omega}^{\dagger}=M \partial_{0} \Omega^{\dagger}-\sigma_{3} M \partial_{r} \Omega^{\dagger}-\sigma_{1} M \frac{1}{r} \partial_{\theta} \Omega^{\dagger}-\sigma_{2} M \frac{1}{r \sin \theta} \partial_{\varphi} \Omega^{\dagger} .
$$

We let $M=\left(\begin{array}{ll}u & v \\ w & s\end{array}\right)$, we then get:

$$
\begin{aligned}
\underline{\nabla}^{\prime} M \underline{\Omega}^{\dagger}= & 0-\sigma_{3} M \partial_{r} \Omega^{\dagger}-\sigma_{1} M \frac{1}{r} \partial_{\theta} \Omega^{\dagger}-\sigma_{2} M \frac{1}{r \sin \theta} \partial_{\varphi} \Omega^{\dagger} \\
= & r^{-2}(\sin \theta)^{-\frac{1}{2}}\left(\begin{array}{cc}
u & v \\
-w & -s
\end{array}\right) S^{\dagger} \\
& +r^{-2}(\sin \theta)^{-\frac{1}{2}}\left(\begin{array}{cc}
-w & -s \\
-u & -v
\end{array}\right)\left(\begin{array}{cc}
-\frac{\cos \theta}{2 \sin \theta} & \frac{1}{2} \\
-\frac{1}{2} & -\frac{\cos \theta}{2 \sin \theta}
\end{array}\right) S^{\dagger}
\end{aligned}
$$




$$
\begin{aligned}
& +r^{-2}(\sin \theta)^{-\frac{3}{2}}\left(\begin{array}{cc}
i w & i s \\
-i u & -i v
\end{array}\right)\left(\begin{array}{cc}
\cos \theta & \sin \theta \\
-\sin \theta & \cos \theta
\end{array}\right)\left(\begin{array}{cc}
\frac{i}{2} & 0 \\
0 & -\frac{i}{2}
\end{array}\right) S^{\dagger} \\
& =r^{-2}(\sin \theta)^{-\frac{1}{2}}\left(\begin{array}{cc}
u+s & v+s \frac{\cos \theta}{\sin \theta} \\
-w+u \frac{\cos \theta}{\sin \theta} & -(s+u)
\end{array}\right) S^{\dagger} .
\end{aligned}
$$

If $M=\hat{X} X^{\dagger}=\hat{X} \sigma_{0} X^{\dagger}$ and since:

$$
\hat{X} X^{\dagger}=\operatorname{det}\left(X^{\dagger}\right)=\rho_{X} \mathrm{e}^{-i \beta}=u=s ; v=w=0,
$$

we get:

$$
\frac{\underline{\nabla}^{\prime} \hat{X} \sigma_{0} X^{\dagger} \underline{\Omega}^{\dagger}}{\operatorname{det}\left(X^{\dagger}\right)}=\left[\frac{2}{r} \sigma_{3}+\frac{\cos \theta}{r \sin \theta} \sigma_{1}\right] \Omega^{\dagger} ; \rho=\frac{\rho_{X}}{r^{2} \sin \theta}=\frac{\left|D \bar{A} U^{2}+B \bar{C} V^{2}\right|}{r^{2} \sin \theta} .
$$

\subsection{Terms with Index 0 and 3}

For the $\sigma_{0}$ case we have:

$$
\begin{aligned}
\frac{\nabla\left(\rho \mathrm{e}^{-i \beta}\right)}{\rho \mathrm{e}^{-i \beta}} & =\frac{(\nabla \rho) \mathrm{e}^{-i \beta}-i(\nabla \beta) \rho \mathrm{e}^{-i \beta}}{\rho \mathrm{e}^{-i \beta}}=\nabla(\ln \rho)-i \nabla \beta \\
& =\frac{\nabla\left(\hat{\phi} \phi^{\dagger}\right)}{\hat{\phi} \phi^{\dagger}}=\frac{\Omega \nabla^{\prime}\left(\hat{X} X^{\dagger} \Omega^{\dagger}\right)}{\Omega \hat{X} X^{\dagger} \Omega^{\dagger}}=\frac{\Omega \nabla^{\prime}\left(\hat{X} X^{\dagger}\right) \Omega^{\dagger}+\Omega \underline{\nabla}^{\prime} \hat{X} X^{\dagger} \underline{\Omega}}{\hat{X} X^{\dagger} \Omega \Omega^{\dagger}} \\
& =S \frac{\nabla^{\prime}\left(\hat{X} X^{\dagger}\right)}{\hat{X} X^{\dagger}} S^{\dagger}+S\left[\frac{2}{r} \sigma_{3}+\frac{\cos \theta}{r \sin \theta} \sigma_{1}\right] S^{\dagger} .
\end{aligned}
$$

Next we have:

$$
\begin{aligned}
\nabla^{\prime}\left(\hat{X} X^{\dagger}\right)= & -\left(\sigma_{3} \partial_{r}+\sigma_{1} \frac{1}{r} \partial_{\theta}\right)\left(D \bar{A} U^{2}+B \bar{C} V^{2}\right) \\
= & -\left(D^{\prime} \bar{A} U^{2}+D \overline{A^{\prime}} U^{2}+B^{\prime} \bar{C} V^{2}+B \bar{C}^{\prime} V^{2}\right) \sigma_{3} \\
& -\frac{2}{r}\left(D \bar{A} U U^{\prime}+B \bar{C} V V^{\prime}\right) \sigma_{1}
\end{aligned}
$$

Using (63) and (64) we obtain:

$$
\begin{gathered}
\bar{A}^{\prime}=-i\left(E+\frac{\alpha}{r}\right) \bar{A}-\frac{\kappa}{r} \bar{C}+i m \mathrm{e}^{-i \beta} \bar{D}, \\
B^{\prime}=i\left(E+\frac{\alpha}{r}\right) B-\frac{\kappa}{r} D-i m \mathrm{e}^{-i \beta} C, \\
\bar{C}^{\prime}=i\left(E+\frac{\alpha}{r}\right) \bar{C}-\frac{\kappa}{r} \bar{A}-i m \mathrm{e}^{-i \beta} \bar{B} \\
D^{\prime}=-i\left(E+\frac{\alpha}{r}\right) D-\frac{\kappa}{r} B+i m \mathrm{e}^{-i \beta} A, \\
U^{\prime}=\frac{\lambda}{\sin \theta} U-\kappa V ; V^{\prime}=-\frac{\lambda}{\sin \theta} V+\kappa U .
\end{gathered}
$$


We let:

$$
\begin{gathered}
r_{1}:=\frac{(B \bar{B}+C \bar{C}) V^{2}-(A \bar{A}+D \bar{D}) U^{2}}{\left|\operatorname{det}\left(X^{\dagger}\right)\right|} ; p_{1}+i p_{2}:=\frac{(D \bar{A}-B \bar{C}) U V}{\operatorname{det}\left(X^{\dagger}\right)} \\
q_{1}+i q_{2}:=\frac{(D \bar{C}+B \bar{A})\left(U^{2}+V^{2}\right)}{\operatorname{det}\left(X^{\dagger}\right)} ; s_{1}+i s_{2}:=\frac{D \bar{A} U^{2}-B \bar{C} V^{2}}{\operatorname{det}\left(X^{\dagger}\right)} .
\end{gathered}
$$

These functions $r_{1}, p_{1}, p_{2}, q_{1}, q_{2}, s_{1}, s_{2}$ depend on $r$ and $\theta$, with values in $\mathbb{R}$. We get:

$$
\begin{aligned}
\frac{\nabla^{\prime}\left(\hat{X} X^{\dagger}\right)}{\hat{X} X^{\dagger}}= & {\left[-2 i\left(E+\frac{\alpha}{r}\right)\left(s_{1}+i s_{2}\right)+\frac{\kappa}{r}\left(q_{1}+i q_{2}\right)+i m r_{1}\right] \sigma_{3} } \\
& +\left[-\frac{2 \lambda}{r \sin \theta}\left(s_{1}+i s_{2}\right)+\frac{2 \kappa}{r}\left(p_{1}+i p_{2}\right)\right] \sigma_{1} .
\end{aligned}
$$

We then get:

$$
\begin{aligned}
S^{\dagger}[\nabla(\ln \rho)-i \nabla \beta] S= & {\left[-\frac{2 \lambda}{r \sin \theta}\left(s_{1}+i s_{2}\right)+\frac{2 \kappa}{r}\left(p_{1}+i p_{2}\right)+\frac{\cos \theta}{r \sin \theta}\right] \sigma_{1} } \\
& +\left[2 i\left(E+\frac{\alpha}{r}\right)\left(s_{1}+i s_{2}\right)+\frac{\kappa}{r}\left(q_{1}+i q_{2}\right)+i m r_{1}+\frac{2}{r}\right] \sigma_{3} .
\end{aligned}
$$

This gives:

$$
\begin{gathered}
\nabla(\ln \rho)=S\left[\left(-\frac{2 \lambda}{r \sin \theta} S_{1}+\frac{2 \kappa}{r} p_{1}+\frac{\cos \theta}{r \sin \theta}\right) \sigma_{1}+\left(\frac{\kappa}{r} q_{1}+\frac{2}{r}\right) \sigma_{3}\right] S^{\dagger} \\
\nabla[\ln (\rho)]=S \overrightarrow{\mathbf{K}}_{0} S^{\dagger} ; \mathbf{K}_{0}:=\mathbf{K}_{0}^{\mu} \sigma_{\mu} ; \mathbf{K}_{0}^{0}=0, \\
\mathbf{K}_{0}^{2}=0 ; \mathbf{K}_{0}^{1}=\frac{\cos \theta-2 \lambda s_{1}}{r \sin \theta}+\frac{2 \kappa}{r} p_{1} ; \mathbf{K}_{0}^{3}=\frac{2}{r}+\frac{\kappa}{r} q_{1}-2\left(E+\frac{\alpha}{r}\right) s_{2} .
\end{gathered}
$$

We let:

$$
\begin{gathered}
r_{2}:=\frac{(A \bar{A}+D \bar{D}) U^{2}+(B \bar{B}+C \bar{C}) V^{2}}{\left|\operatorname{det}\left(X^{\dagger}\right)\right|} ; r_{3}:=\frac{\Re(D \bar{B}-A \bar{C}) U V}{\left|\operatorname{det}\left(X^{\dagger}\right)\right|}, \\
r_{4}:=\frac{\Im(B \bar{D}-C \bar{A}) U V}{\left|\operatorname{det}\left(X^{\dagger}\right)\right|} ; v_{1}+i v_{2}:=\frac{(D \bar{C}-B \bar{A})\left(U^{2}-V^{2}\right)}{\operatorname{det}\left(X^{\dagger}\right)}, \\
t_{1}+i t_{2}:=\frac{(D \bar{C}-B \bar{A}) U V}{\operatorname{det}\left(X^{\dagger}\right)} ; u_{1}+i u_{2}:=\frac{(D \bar{C}+B \bar{A}) U V}{\operatorname{det}\left(X^{\dagger}\right)}
\end{gathered}
$$

where $r_{2}, r_{3}, r_{4}, v_{1}, v_{2}, t_{1}, t_{2}, u_{1}, u_{2}$ depend on $r$ and $\theta$, with values in $\mathbb{R}$. The $\sigma_{3}$ case gives:

$$
\begin{gathered}
M:=\hat{X} \sigma_{3} X^{\dagger}=\left(\begin{array}{cc}
L_{1} \bar{R}_{1}-L_{2} \bar{R}_{2} & 2 L_{1} \bar{R}_{2} \\
2 L_{2} \bar{R}_{1} & -L_{1} \bar{R}_{1}+L_{2} \bar{R}_{2}
\end{array}\right), \\
u=L_{1} \bar{R}_{1}-L_{2} \bar{R}_{2} ; v=2 L_{1} \bar{R}_{2} ; w=2 L_{2} \bar{R}_{1} ; s=-u,
\end{gathered}
$$

Using (72) we get: 


$$
\begin{gathered}
\underline{\nabla}^{\prime} M \underline{\Omega}^{\dagger}=r^{-2}(\sin \theta)^{-\frac{1}{2}}\left(\begin{array}{cc}
0 & v-u \frac{\cos \theta}{\sin \theta} \\
-w+u \frac{\cos \theta}{\sin \theta} & 0
\end{array}\right) S^{\dagger} \\
\frac{\Omega \underline{\nabla}^{\prime} M \underline{\Omega}^{\dagger}}{\operatorname{det}\left(\phi^{\dagger}\right)}=S \frac{1}{r}\left[\begin{array}{c}
+\frac{L_{1} \bar{R}_{2}-L_{2} \bar{R}_{1}}{L_{1} \bar{R}_{1}+L_{2} \bar{R}_{2}} \sigma_{1} \\
+\frac{L_{1} \bar{R}_{2}+L_{2} \bar{R}_{1}}{L_{1} \bar{R}_{1}+L_{2} \bar{R}_{2}} i \sigma_{2}+\frac{L_{2} \bar{R}_{2}-L_{1} \bar{R}_{1}}{L_{1} \bar{R}_{1}+L_{2} \bar{R}_{2}} \frac{\cos \theta}{\sin \theta} i \sigma_{2}
\end{array}\right] S^{\dagger} \\
=S \frac{1}{r}\left[\left(t_{1}+i t_{2}\right) \sigma_{1}+\left(u_{1}+i u_{2}\right) i \sigma_{2}-\left(s_{1}+i s_{2}\right) \frac{\cos \theta}{\sin \theta} i \sigma_{2}\right] S^{\dagger} .
\end{gathered}
$$

Next we have the following, with the radial and angular functions previously defined in (61):

$$
\begin{aligned}
\nabla^{\prime}\left[\hat{X} \sigma_{3} X^{\dagger}\right]= & \left(\begin{array}{cc}
-\partial_{r} & -\frac{1}{r} \partial_{\theta} \\
-\frac{1}{r} \partial_{\theta} & \partial_{r}
\end{array}\right)\left(\begin{array}{cc}
D \bar{A} U^{2}-B \bar{C} V^{2} & 2 D \bar{C} U V \\
2 B \bar{A} U V & B \bar{C} V^{2}-D \bar{A} U^{2}
\end{array}\right) \\
= & {\left[-(D \bar{A})^{\prime} U^{2}+(B \bar{C})^{\prime} V^{2}-\frac{2}{r} B \bar{A}(U V)^{\prime}\right] \frac{1+\sigma_{3}}{2} } \\
& +\left[-(D \bar{A})^{\prime} U^{2}+(B \bar{C})^{\prime} V^{2}-\frac{2}{r} D \bar{C}(U V)^{\prime}\right] \frac{1-\sigma_{3}}{2} \\
& {\left[-\frac{D \bar{A}}{r} U U^{\prime}+\frac{B \bar{C}}{r} V V^{\prime}+(B \bar{A})^{\prime} U V\right]\left(\sigma_{1}-i \sigma_{2}\right) } \\
& +\left[+\frac{D \bar{A}}{r} U U^{\prime}-\frac{B \bar{C}}{r} V V^{\prime}-(D \bar{C})^{\prime} U V\right]\left(\sigma_{1}+i \sigma_{2}\right) . \\
\nabla^{\prime}\left(\hat{X} \sigma_{3} X^{\dagger}\right)= & -(D \bar{A})^{\prime} U^{2}+(B \bar{C})^{\prime} V^{2}-\frac{B \bar{A}+D \bar{C}}{r}(U V)^{\prime} \\
& +\frac{1}{r}(D \bar{C}-B \bar{A})(U V)^{\prime} \sigma_{3}+(B \bar{A}-D \bar{C})^{\prime} U V \sigma_{1} \\
& +\left[\frac{2}{r}\left(D \bar{A} U U^{\prime}-B \bar{C} V V^{\prime}\right)-(B \bar{A}+D \bar{C})^{\prime} U V\right] i \sigma_{2} .
\end{aligned}
$$

And we get, using (77) and (78):

$$
\begin{aligned}
\frac{\nabla^{\prime}\left(\hat{X} \sigma_{3} X^{\dagger}\right)}{\hat{X} X^{\dagger}}= & 2 i\left(E+\frac{\alpha}{r}\right)-i m r_{2}+\frac{\kappa}{r}\left(v_{1}+i v_{2}\right) \sigma_{3}+2 m r_{3} \sigma_{2} \\
& +\left(\frac{2 \lambda}{r \sin \theta}+2 m r_{4}\right) i \sigma_{2} .
\end{aligned}
$$

We then get:

$$
\begin{aligned}
S^{\dagger} \frac{\nabla\left(\hat{\phi} \sigma_{3} \phi^{\dagger}\right)}{\operatorname{det}\left(\phi^{\dagger}\right)} S= & 2 i\left(E+\frac{\alpha}{r}\right)-i m r_{2}+\frac{t_{1}+i t_{2}}{r} \sigma_{1}+2 m r_{3} \sigma_{2} \\
& +\left[2 i m r_{4}+\frac{2 i \lambda}{r \sin \theta}+\frac{i u_{1}-u_{2}}{r}+\frac{\left(s_{2}-i s_{1}\right) \cos \theta}{r \sin \theta}\right] \sigma_{2} \\
& +\frac{\kappa}{r}\left(v_{1}+i v_{2}\right) \sigma_{3},
\end{aligned}
$$




$$
\begin{aligned}
& \mathcal{S}_{(3)}=\frac{1}{2}\left[\frac{\nabla\left(\hat{\phi} \sigma_{3} \phi^{\dagger}\right)}{\operatorname{det}\left(\phi^{\dagger}\right)}+\left(\frac{\nabla\left(\hat{\phi} \sigma_{3} \phi^{\dagger}\right)}{\operatorname{det}\left(\phi^{\dagger}\right)}\right)^{\dagger}\right]=S \mathbf{K}_{3} S^{\dagger} \\
&=S\left[\frac{t_{1}}{r} \sigma_{1}+\left(-\frac{u_{2}}{r}+\frac{S_{2} \cos \theta}{r \sin \theta}\right) \sigma_{2}+\frac{\kappa}{r} v_{1} \sigma_{3}\right] S^{\dagger} \\
& \mathbf{K}_{3}^{0}=0 ; \mathbf{K}_{3}^{1}=\frac{t_{1}}{r} ; \mathbf{K}_{3}^{2}=2 m r_{3}-\frac{u_{2}}{r}+\frac{S_{2}}{r} \frac{\cos \theta}{\sin \theta} ; \mathbf{K}_{3}^{3}=\frac{\kappa}{r} v_{1} .
\end{aligned}
$$

\subsection{Calculation of the Currents}

We have:

$$
D_{\mu}=\phi \sigma_{\mu} \phi^{\dagger}=\Omega X \mathrm{e}^{\zeta i_{3}} \sigma_{\mu} \mathrm{e}^{-\zeta i_{3}} X^{\dagger} \Omega^{\dagger}=S \frac{X \mathrm{e}^{\zeta i_{3}} \sigma_{\mu} \mathrm{e}^{-\zeta i_{3}} X^{\dagger}}{r^{2} \sin \theta} S^{\dagger} .
$$

We then let, for any space-time vector $\mathcal{V}$ :

$$
\mathbf{V}:=S^{\dagger} \mathcal{V} S ; \mathrm{d}_{\mu}:=X \mathrm{e}^{\zeta i_{3}} \sigma_{\mu} \mathrm{e}^{-\zeta i_{3}} X^{\dagger} .
$$

We then have:

$$
\begin{aligned}
2 D_{\mu} \cdot \mathcal{V} & =D_{\mu} \hat{\mathcal{V}}+\mathcal{V} \hat{D}_{\mu}=S \mathrm{~d}_{\mu} S^{\dagger} S \hat{\mathbf{V}} S^{\dagger}+S \mathbf{V} S^{\dagger} S \hat{\mathrm{d}}_{\mu} S^{\dagger} \\
& =S\left(\mathrm{~d}_{\mu} \hat{\mathbf{V}}+\mathbf{V} \hat{\mathrm{d}}_{\mu}\right) S^{\dagger}=S\left(2 \mathrm{~d}_{\mu} \cdot \mathbf{V}\right) S^{\dagger}=2 \mathrm{~d}_{\mu} \cdot \mathbf{V} S S^{\dagger}=2 \mathrm{~d}_{\mu} \cdot \mathbf{V} .
\end{aligned}
$$

This allows us a simplification of the scalar product. We get:

$$
\begin{gathered}
\mathrm{d}_{0}=X X^{\dagger}=\left(\begin{array}{ll}
R_{1} \bar{R}_{1}+L_{2} \bar{L}_{2} & R_{1} \bar{R}_{2}-L_{1} \bar{L}_{2} \\
R_{2} \bar{R}_{1}-L_{2} \bar{L}_{1} & L_{1} \bar{L}_{1}+R_{2} \bar{R}_{2}
\end{array}\right), \\
\mathrm{d}_{0}^{0}+\mathrm{d}_{0}^{3}=R_{1} \bar{R}_{1}+L_{2} \bar{L}_{2} ; \mathrm{d}_{0}^{1}-i \mathrm{~d}_{0}^{2}=R_{1} \bar{R}_{2}-L_{1} \bar{L}_{2}, \\
\mathrm{~d}_{0}^{0}-\mathrm{d}_{0}^{3}=L_{1} \bar{L}_{1}+R_{2} \bar{R}_{2} ; \mathrm{d}_{0}^{1}+i \mathrm{~d}_{0}^{2}=R_{2} \bar{R}_{1}-L_{2} \bar{L}_{1} .
\end{gathered}
$$

Similarly we have:

$$
\begin{aligned}
& \mathrm{d}_{3}=X \sigma_{3} X^{\dagger}=\left(\begin{array}{cc}
R_{1} \bar{R}_{1}-L_{2} \bar{L}_{2} & R_{1} \bar{R}_{2}+L_{1} \bar{L}_{2} \\
R_{2} \bar{R}_{1}+L_{2} \bar{L}_{1} & -L_{1} \bar{L}_{1}+R_{2} \bar{R}_{2}
\end{array}\right), \\
& \mathrm{d}_{3}^{0}+\mathrm{d}_{3}^{3}=R_{1} \bar{R}_{1}-L_{2} \bar{L}_{2} ; \mathrm{d}_{3}^{1}-i \mathrm{~d}_{3}^{2}=R_{1} \bar{R}_{2}+L_{1} \bar{L}_{2}, \\
& \mathrm{~d}_{3}^{0}-\mathrm{d}_{3}^{3}=-L_{1} \bar{L}_{1}+R_{2} \bar{R}_{2} ; \mathrm{d}_{3}^{1}+i \mathrm{~d}_{3}^{2}=R_{2} \bar{R}_{1}+L_{2} \bar{L}_{1} .
\end{aligned}
$$

Sum and difference of $d_{0}$ and $d_{3}$ are simple, which will be useful. We get:

$$
\begin{gathered}
\mathrm{d}_{0}+\mathrm{d}_{3}=X\left(1+\sigma_{3}\right) X^{\dagger}=2\left(\begin{array}{cc}
R_{1} \bar{R}_{1} & R_{1} \bar{R}_{2} \\
R_{2} \bar{R}_{1} & R_{2} \bar{R}_{2}
\end{array}\right), \\
\mathrm{d}_{0}-\mathrm{d}_{3}=X\left(1-\sigma_{3}\right) X^{\dagger}=2\left(\begin{array}{cc}
L_{2} \bar{L}_{2} & -L_{1} \bar{L}_{2} \\
-L_{2} \bar{L}_{1} & L_{1} \bar{L}_{1}
\end{array}\right) .
\end{gathered}
$$

Next we have:

$$
\mathrm{d}_{1}+i \mathrm{~d}_{2}=X \mathrm{e}^{2 \zeta i_{3}}\left(\sigma_{1}+i \sigma_{2}\right) X^{\dagger}=2 \mathrm{e}^{2 i \zeta}\left(\begin{array}{ll}
-R_{1} L_{2} & R_{1} L_{1} \\
-R_{2} L_{2} & R_{2} L_{1}
\end{array}\right),
$$




$$
\mathrm{d}_{1}-i \mathrm{~d}_{2}=X \mathrm{e}^{2 \zeta i_{3}}\left(\sigma_{1}-i \sigma_{2}\right) X^{\dagger}=2 \mathrm{e}^{-2 i \zeta}\left(\begin{array}{cc}
\bar{L}_{2} \bar{R}_{1} & -\bar{L}_{2} \bar{R}_{2} \\
\bar{L}_{1} \bar{R}_{1} & \bar{L}_{1} \bar{R}_{2}
\end{array}\right)
$$

\subsection{Calculation of 12 Christoffel Symbols}

We have:

$$
\Gamma_{0 \mu}^{0}=D_{\mu} \cdot \nabla(\ln \rho)=\mathrm{d}_{\mu} \cdot \mathbf{K}_{0} ; \Gamma_{3 \mu}^{0}=D_{\mu} \cdot \mathcal{S}_{(3)}=\mathrm{d}_{\mu} \cdot \mathbf{K}_{3} .
$$

We also get the symbols:

$$
\begin{aligned}
\Gamma_{30}^{0}+\Gamma_{33}^{0}=- & \left(\mathrm{d}_{0}^{1}+\mathrm{d}_{3}^{1}\right) \mathbf{K}_{3}^{1}-\left(\mathrm{d}_{0}^{2}+\mathrm{d}_{3}^{2}\right) \mathbf{K}_{3}^{2}-\left(\mathrm{d}_{0}^{3}+\mathrm{d}_{3}^{3}\right) \mathbf{K}_{3}^{3} \\
=- & \left(R_{1} \bar{R}_{2}+R_{2} \bar{R}_{1}\right) \mathbf{K}_{3}^{1}-2 \Im\left(R_{1} \bar{R}_{2}\right) \mathbf{K}_{3}^{2}-\left(R_{1} \bar{R}_{1}-R_{2} \bar{R}_{2}\right) \mathbf{K}_{3}^{3}, \\
\Gamma_{30}^{0}-\Gamma_{33}^{0}=- & -\left(\mathrm{d}_{0}^{1}-\mathrm{d}_{3}^{1}\right) \mathbf{K}_{3}^{1}-\left(\mathrm{d}_{0}^{2}-\mathrm{d}_{3}^{2}\right) \mathbf{K}_{3}^{2}-\left(\mathrm{d}_{0}^{3}-\mathrm{d}_{3}^{3}\right) \mathbf{K}_{3}^{3} \\
= & \left(L_{1} \bar{L}_{2}+L_{2} \bar{L}_{1}\right) \mathbf{K}_{3}^{1}+2 \Im\left(L_{2} \bar{L}_{1}\right) \mathbf{K}_{3}^{2}+\left(L_{1} \bar{L}_{1}-L_{2} \bar{L}_{2}\right) \mathbf{K}_{3}^{3} \\
\Gamma_{31}^{0}+i \Gamma_{32}^{0}= & -\left(\mathrm{d}_{1}^{1}+i \mathrm{~d}_{2}^{1}\right) \mathbf{K}_{3}^{1}-\left(\mathrm{d}_{1}^{2}+i \mathrm{~d}_{2}^{2}\right) \mathbf{K}_{3}^{2}-\left(\mathrm{d}_{1}^{3}+i \mathrm{~d}_{2}^{3}\right) \mathbf{K}_{3}^{3} \\
= & -\mathrm{e}^{2 i \zeta}\left(R_{1} L_{1}-R_{2} L_{2}\right) \mathbf{K}_{3}^{1}+i \mathrm{e}^{2 i \zeta}\left(R_{1} L_{1}+R_{2} L_{2}\right) \mathbf{K}_{3}^{2} \\
& +\mathrm{e}^{2 i \zeta}\left(R_{1} L_{2}-R_{2} L_{1}\right) \mathbf{K}_{3}^{3} .
\end{aligned}
$$

We obtain also:

$$
\begin{gathered}
\mathbf{K}_{3}^{\prime}=S^{\dagger}\left(\mathcal{S}_{(3)}^{\prime}+2 q A\right) S=\frac{S^{\dagger}}{2 i}\left[\frac{\nabla\left(\hat{\phi} \sigma_{3} \phi^{\dagger}\right)}{\operatorname{det}\left(\phi^{\dagger}\right)}-\left(\frac{\nabla\left(\hat{\phi} \sigma_{3} \phi^{\dagger}\right)}{\operatorname{det}\left(\phi^{\dagger}\right)}\right)^{\dagger}-2 i \frac{\alpha}{r}\right] S \\
=2 E-m r_{2}+\left(2 m r_{3}+\frac{t_{2}}{r}\right) \sigma_{1}+\left(2 m r_{4}+\frac{2 \lambda-s_{1} \cos \theta}{r \sin \theta}+\frac{u_{1}}{r}\right) \sigma_{2}+\frac{\kappa}{r} v_{2} \sigma_{3} \\
\mathbf{K}_{3}^{\prime 0}=2 E-m r_{2} ; \mathbf{K}_{3}^{\prime 1}=2 m r_{3}+\frac{t_{2}}{r} \\
\mathbf{K}_{3}^{\prime 2}=2 m r_{4}+\frac{2 \lambda}{r \sin \theta}+\frac{u_{1}}{r}-\frac{s_{1}}{r} \frac{\cos \theta}{\sin \theta} ; \mathbf{K}_{3}^{\prime 3}=\frac{\kappa}{r} v_{2} .
\end{gathered}
$$

We then get from (15):

$$
\begin{aligned}
& \Gamma_{2 \mu}^{1}=-2 m \rho \delta_{\mu}^{0}-\mathrm{d}_{\mu} \cdot \mathbf{K}_{3}^{\prime}, \\
& \Gamma_{20}^{1}=-2 m \rho-\mathrm{d}_{0} \cdot \mathbf{K}_{3}^{\prime} \\
&=-2 m \rho-\frac{1}{2}\left(R_{1} \bar{R}_{1}+R_{2} \bar{R}_{2}+L_{1} \bar{L}_{1}+L_{2} \bar{L}_{2}\right) \mathbf{K}_{3}^{\prime 0} \\
&+\Re\left(R_{1} \bar{R}_{2}-L_{1} \bar{L}_{2}\right) \mathbf{K}_{3}^{\prime 1}+\Im\left(R_{1} \bar{R}_{2}+L_{1} \bar{L}_{2}\right) \mathbf{K}_{3}^{\prime 2} \\
&+\frac{1}{2}\left(R_{1} \bar{R}_{1}-R_{2} \bar{R}_{2}-L_{1} \bar{L}_{1}+L_{2} \bar{L}_{2}\right) \mathbf{K}_{3}^{\prime 3}, \\
& \Gamma_{23}^{1}=-\mathrm{d}_{3} \cdot \mathbf{K}_{3}^{\prime}=-\frac{1}{2}\left(R_{1} \bar{R}_{1}-R_{2} \bar{R}_{2}-L_{1} \bar{L}_{1}+L_{2} \bar{L}_{2}\right) \mathbf{K}_{3}^{\prime 0} \\
&+ \Re\left(R_{1} \bar{R}_{2}+L_{1} \bar{L}_{2}\right) \mathbf{K}_{3}^{\prime 1}+\Im\left(R_{1} \bar{R}_{2}+L_{1} \bar{L}_{2}\right) \mathbf{K}_{3}^{\prime 2} \\
&+ \frac{1}{2}\left(R_{1} \bar{R}_{1}-R_{2} \bar{R}_{2}-L_{1} \bar{L}_{1}+L_{2} \bar{L}_{2}\right) \mathbf{K}_{3}^{\prime 0},
\end{aligned}
$$




$$
\begin{aligned}
\Gamma_{21}^{1}+i \Gamma_{22}^{1}= & -\left(\mathrm{d}_{1} \cdot \mathbf{K}_{3}^{\prime}+i \mathrm{~d}_{2} \cdot \mathbf{K}_{3}^{\prime}\right) \\
= & \mathrm{e}^{2 i \zeta}\left(-R_{1} L_{2}+R_{2} L_{1}\right) \mathbf{K}_{3}^{\prime 0}+\mathrm{e}^{2 i \zeta}\left(R_{1} L_{1}-R_{2} L_{2}\right) \mathbf{K}_{3}^{\prime 1} \\
& -i \mathrm{e}^{2 i \zeta}\left(R_{1} L_{1}+R_{2} L_{2}\right) \mathbf{K}_{3}^{\prime 2}+\mathrm{e}^{2 i \zeta}\left(-R_{1} L_{2}-R_{2} L_{1}\right) \mathbf{K}_{3}^{\prime 3} .
\end{aligned}
$$

\subsection{Terms with Index 1 and 2}

Now we consider:

$$
M^{+}:=\hat{X} \mathrm{e}^{2 \zeta i_{3}}\left(\sigma_{1}+i \sigma_{2}\right) X^{\dagger} ; M^{-}:=\hat{X} \mathrm{e}^{2 \zeta i_{3}}\left(\sigma_{1}-i \sigma_{2}\right) X^{\dagger},
$$

This gives:

$$
\begin{gathered}
\underline{\nabla}^{\prime} M^{+} \underline{\Omega}^{\dagger}=\frac{\mathrm{e}^{2 i \zeta}}{r^{2} \sqrt{\sin \theta}}\left[\left(L_{1}^{2}+L_{2}^{2}\right) \sigma_{1}+i\left(L_{1}^{2}-L_{2}^{2}+2 L_{1} L_{2} \frac{\cos \theta}{\sin \theta}\right) \sigma_{2}\right] S^{\dagger} \\
\underline{\nabla}^{\prime} M^{-} \underline{\Omega}^{\dagger}=\frac{\mathrm{e}^{-2 i \zeta}}{r^{2} \sqrt{\sin \theta}}\left[-\left(\bar{R}_{1}^{2}+\bar{R}_{2}^{2}\right) \sigma_{1}+i\left(\bar{R}_{1}^{2}-\bar{R}_{2}^{2}+2 \bar{R}_{1} \bar{R}_{2} \frac{\cos \theta}{\sin \theta}\right) \sigma_{2}\right] S^{\dagger} .
\end{gathered}
$$

Then we have:

$$
\begin{aligned}
& \frac{\nabla\left[\hat{\phi}\left(\sigma_{1}+i \sigma_{2}\right) \phi^{\dagger}\right]}{\hat{\phi} \phi^{\dagger}}=\frac{\Omega \nabla^{\prime}\left[\hat{X} \mathrm{e}^{\zeta i_{3}}\left(\sigma_{1}+i \sigma_{2}\right) \mathrm{e}^{-\zeta i_{3}} X^{\dagger} \Omega^{\dagger}\right]}{\Omega \hat{X} X^{\dagger} \Omega^{\dagger}} \\
= & \frac{\Omega \nabla^{\prime}\left[\hat{X} \mathrm{e}^{2 \zeta i_{3}}\left(\sigma_{1}+i \sigma_{2}\right) X^{\dagger}\right] \Omega^{\dagger}}{\Omega \hat{X} X^{\dagger} \Omega^{\dagger}}+\frac{\Omega \nabla^{\prime} \hat{X} \mathrm{e}^{2 \zeta i_{3}}\left(\sigma_{1}+i \sigma_{2} X^{\dagger}\right) \underline{\Omega}^{\dagger}}{\Omega \hat{X} X^{\dagger} \Omega^{\dagger}} \\
= & S\left[\frac{\nabla^{\prime}\left[\hat{X} \mathrm{e}^{2 \zeta i_{3}}\left(\sigma_{1}+i \sigma_{2}\right) X^{\dagger}\right]}{\hat{X} X^{\dagger}}\right. \\
& \left.+\frac{2 \mathrm{e}^{2 i \zeta} \frac{1}{r}\left[\left(L_{1}^{2}+L_{2}^{2}\right) \sigma_{1}+\left(L_{1}^{2}-L_{2}^{2}+2 L_{1} L_{2} \frac{\cos \theta}{\left.\sin \theta) i \sigma_{2}\right]}\right.\right.}{\hat{X} X^{\dagger}}\right] S^{\dagger} .
\end{aligned}
$$

So we get:

$$
\begin{aligned}
& \frac{\nabla\left(S_{1}^{\dagger}+i S_{2}^{\dagger}\right)+2 i q A\left(S_{1}^{\dagger}+i S_{2}^{\dagger}\right)}{\operatorname{det}\left(\phi^{\dagger}\right)}=\frac{\nabla\left[\hat{\phi}\left(\sigma_{1}+i \sigma_{2}\right) \phi^{\dagger}\right]+2 i q A \hat{\phi}\left(\sigma_{1}+i \sigma_{2}\right) \phi^{\dagger}}{\hat{\phi} \phi^{\dagger}} \\
& =S\left[\frac{\nabla^{\prime} M^{+}-2 i \frac{\alpha}{r} M^{+}}{\hat{X} X^{\dagger}}+\frac{2 \mathrm{e}^{2 i \zeta} \frac{1}{r}\left[\left(L_{1}^{2}+L_{2}^{2}\right) \sigma_{1}+\left(L_{1}^{2}-L_{2}^{2}+2 L_{1} L_{2} \frac{\cos \theta}{\sin \theta}\right) i \sigma_{2}\right]}{\hat{X} X^{\dagger}}\right] S^{\dagger} .
\end{aligned}
$$

We calculate now:

$$
\begin{aligned}
& \nabla^{\prime} M^{+}+2 i q A M^{+} \\
& =\left(\partial_{0}-\sigma_{3} \partial_{r}-\sigma_{1} \frac{1}{r} \partial_{\theta}-\sigma_{2} \frac{1}{r \sin \theta} \partial_{\varphi}\right)\left[\hat{X} \mathrm{e}^{2 \zeta i_{3}}\left(\sigma_{1}+i \sigma_{2}\right) X^{\dagger}\right]-2 i \frac{\alpha}{r} M^{+} \\
& =\left(\begin{array}{ll}
\mathcal{A} & \mathcal{C} \\
\mathcal{B} & \mathcal{D}
\end{array}\right)-4 i \frac{\alpha}{r} \mathrm{e}^{2 i \zeta}\left(\begin{array}{cc}
-B D U V & D^{2} U^{2} \\
-B^{2} V^{2} & B D U V
\end{array}\right)
\end{aligned}
$$




$$
\begin{gathered}
\mathcal{A}=\left(\partial_{0}-\partial_{r}\right)\left(-2 \mathrm{e}^{2 i \zeta} D B U V\right)+\left(-\frac{1}{r} \partial_{\theta}+\frac{i}{r \sin \theta} \partial_{\varphi}\right)\left(-2 \mathrm{e}^{2 i \zeta} B^{2} V^{2}\right), \\
\mathcal{B}=\left(\partial_{0}+\partial_{r}\right)\left(-2 \mathrm{e}^{2 i \zeta} B^{2} V^{2}\right)+\left(-\frac{1}{r} \partial_{\theta}+\frac{i}{r \sin \theta} \partial_{\varphi}\right)\left(-2 \mathrm{e}^{2 i \zeta} D B U V\right), \\
\mathcal{C}=\left(\partial_{0}-\partial_{r}\right)\left(2 \mathrm{e}^{2 i \zeta} D^{2} U^{2}\right)+\left(-\frac{1}{r} \partial_{\theta}+\frac{i}{r \sin \theta} \partial_{\varphi}\right)\left(2 \mathrm{e}^{2 i \zeta} D B U V\right), \\
\mathcal{D}=\left(\partial_{0}+\partial_{r}\right)\left(2 \mathrm{e}^{2 i \zeta} D B U V\right)+\left(-\frac{1}{r} \partial_{\theta}-\frac{i}{r \sin \theta} \partial_{\varphi}\right)\left(2 \mathrm{e}^{2 i \zeta} D^{2} U^{2}\right) .
\end{gathered}
$$

This gives:

$$
\begin{gathered}
\frac{\nabla\left[\hat{\phi}\left(\sigma_{1}+i \sigma_{2}\right) \phi^{\dagger}\right]}{\operatorname{det}\left(\phi^{\dagger}\right)}+2 i q A \frac{\hat{\phi}\left(\sigma_{1}+i \sigma_{2}\right) \phi^{\dagger}}{\operatorname{det}\left(\phi^{\dagger}\right)}=S\left(\begin{array}{ll}
\mathcal{A}^{\prime} & \mathcal{C}^{\prime} \\
\mathcal{B}^{\prime} & \mathcal{D}^{\prime}
\end{array}\right) S^{\dagger} \\
\mathcal{A}^{\prime}=\frac{2 \mathrm{e}^{2 i \zeta} U V}{\operatorname{det}\left(X^{\dagger}\right)}\left[\begin{array}{c}
\left.2 i\left(E+\frac{\alpha}{r}\right) D B+i m \mathrm{e}^{-i \beta}(A B-C D)\right] \\
+\frac{\kappa}{r}\left(B^{2}-D^{2}\right)
\end{array}\right], \\
\mathcal{D}^{\prime}=\frac{2 \mathrm{e}^{2 i \zeta} U V}{\operatorname{det}\left(X^{\dagger}\right)}\left[\begin{array}{c}
-2 i\left(E+\frac{\alpha}{r}\right) D B+i m \mathrm{e}^{-i \beta}(A B-C D) \\
-\frac{\kappa}{r}\left(B^{2}-D^{2}\right)
\end{array}\right], \\
\mathcal{B}^{\prime}=\frac{2 \mathrm{e}^{2 i \zeta}}{\operatorname{det}\left(X^{\dagger}\right)}\left[\begin{array}{c}
\frac{B^{2} V^{2}}{r}+2 i m \mathrm{e}^{-i \beta} B C V^{2}+\frac{\kappa}{r} B D\left(U^{2}+V^{2}\right) \\
-\frac{2 \lambda+\cos \theta}{r \sin \theta} B D U V
\end{array}\right], \\
\mathcal{C}^{\prime}=\frac{2 e^{2 i \zeta}}{\operatorname{det}\left(X^{\dagger}\right)}\left[\begin{array}{c}
\frac{D^{2} U^{2}}{r}-2 i m \mathrm{e}^{-i \beta} A D U^{2}+\frac{\kappa}{r} B D\left(U^{2}+V^{2}\right) \\
-\frac{2 \lambda-\cos \theta}{r \sin \theta} B D U V
\end{array}\right] .
\end{gathered}
$$

Similarly we have:

$$
\begin{aligned}
& \frac{\nabla\left[\hat{\phi}\left(\sigma_{1}-i \sigma_{2}\right) \phi^{\dagger}\right]}{\hat{\phi} \phi^{\dagger}}=S\left[\frac{\nabla^{\prime}\left[\hat{X} \mathrm{e}^{2 \zeta i_{3}}\left(\sigma_{1}-i \sigma_{2}\right) X^{\dagger}\right]}{\hat{X} X^{\dagger}}\right. \\
& \left.+\frac{\mathrm{e}^{-2 i \zeta}\left[-\left(\bar{R}_{1}^{2}+\bar{R}_{2}^{2}\right) \sigma_{1}+i\left(\bar{R}_{1}^{2}-\bar{R}_{2}^{2}+2 \bar{R}_{1} \bar{R}_{2} \frac{\cos \theta}{\sin \theta}\right) \sigma_{2}\right]}{\hat{X} X^{\dagger}}\right] S^{\dagger} .
\end{aligned}
$$

We calculate now:

$$
\nabla^{\prime} M^{-}-2 i q A M^{-}=\left(\begin{array}{ll}
\mathcal{A}_{-} & \mathcal{C}_{-} \\
\mathcal{B}_{-} & \mathcal{D}_{-}
\end{array}\right)+4 i \frac{\alpha}{r} \mathrm{e}^{-2 i \zeta}\left(\begin{array}{cc}
-\bar{A} \bar{C} U V & -\bar{C}^{2} U^{2} \\
\bar{A}^{2} V^{2} & \bar{A} \bar{C} U V
\end{array}\right)
$$




$$
\begin{gathered}
\mathcal{A}_{-}=\left(\partial_{0}-\partial_{r}\right)\left(-2 \mathrm{e}^{-2 i \zeta} \bar{A} \bar{C} U V\right)-\left(\frac{1}{r} \partial_{\theta}-\frac{i}{r \sin \theta} \partial_{\varphi}\right)\left(2 \mathrm{e}^{-2 i \zeta} \bar{A}^{2} U^{2}\right), \\
\mathcal{B}_{-}=\left(\partial_{0}+\partial_{r}\right)\left(2 \mathrm{e}^{-2 i \zeta} \bar{A}^{2} U^{2}\right)+\left(\frac{1}{r} \partial_{\theta}+\frac{i}{r \sin \theta} \partial_{\varphi}\right)\left(2 \mathrm{e}^{-2 i \zeta} \bar{A} \bar{C} U V\right), \\
\mathcal{C}_{-}=\left(\partial_{0}-\partial_{r}\right)\left(-2 \mathrm{e}^{-2 i \zeta} \bar{C}^{2} V^{2}\right)+\left(-\frac{1}{r} \partial_{\theta}+\frac{i}{r \sin \theta} \partial_{\varphi}\right)\left(2 \mathrm{e}^{-2 i \zeta} \bar{A} \bar{C} U V\right), \\
\mathcal{D}_{-}=\left(\partial_{0}+\partial_{r}\right)\left(2 \mathrm{e}^{-2 i \zeta} \bar{A} \bar{C} U V\right)+\left(\frac{1}{r} \partial_{\theta}+\frac{i}{r \sin \theta} \partial_{\varphi}\right)\left(2 \mathrm{e}^{-2 i \zeta} \bar{C}^{2} V^{2}\right) .
\end{gathered}
$$

This gives:

$$
\begin{gathered}
\frac{\nabla\left[\hat{\phi}\left(\sigma_{1}-i \sigma_{2}\right) \phi^{\dagger}\right]}{\operatorname{det}\left(\phi^{\dagger}\right)}-2 i q A \frac{\hat{\phi}\left(\sigma_{1}-i \sigma_{2}\right) \phi^{\dagger}}{\operatorname{det}\left(\phi^{\dagger}\right)}=S\left(\begin{array}{ll}
\mathcal{A}_{-}^{\prime} & \mathcal{C}_{-}^{\prime} \\
\mathcal{B}_{-}^{\prime} & \mathcal{D}_{-}^{\prime}
\end{array}\right) S^{\dagger} \\
\mathcal{A}_{-}^{\prime}=\frac{2 \mathrm{e}^{-2 i \zeta} U V}{\operatorname{det}\left(X^{\dagger}\right)}\left[\begin{array}{c}
\left.-2 i\left(E+\frac{\alpha}{r}\right) \bar{A} \bar{C}+i m \mathrm{e}^{-i \beta}(\bar{D} \bar{C}-\bar{A} \bar{B})\right] \\
\frac{\kappa}{r}\left(\bar{A}^{2}-\bar{C}^{2}\right)
\end{array}\right], \\
\mathcal{D}_{-}^{\prime}=\frac{2 \mathrm{e}^{2 i \zeta} U V}{\operatorname{det}\left(X^{\dagger}\right)}\left[\begin{array}{c}
\left.2 i\left(E+\frac{\alpha}{r}\right) \bar{A} \bar{C}-\frac{\kappa}{r}\left(\bar{A}^{2}-\bar{C}^{2}\right)\right] \\
+i m \mathrm{e}^{-i \beta}(\bar{D} \bar{C}-\bar{A} \bar{B})
\end{array}\right], \\
\mathcal{B}_{-}^{\prime}=\frac{2 \mathrm{e}^{-2 i \zeta}}{\operatorname{det}\left(X^{\dagger}\right)}\left[\begin{array}{c}
-\frac{\kappa}{r} \bar{A} \bar{C}\left(U^{2}+V^{2}\right)+2 i m \mathrm{e}^{-i \beta} \bar{D} \bar{A} U^{2} \\
-\frac{\bar{A}^{2} U^{2}}{r}+\frac{2 \lambda-\cos \theta}{r \sin \theta} \bar{A} \bar{C} U V
\end{array}\right], \\
\mathcal{C}_{-}^{\prime}=\frac{2 \mathrm{e}^{-2 i \zeta}}{\operatorname{det}\left(X^{\dagger}\right)}\left[\begin{array}{c}
-\frac{\kappa}{r} \bar{A} \bar{C}\left(U^{2}+V^{2}\right)+2 i m \mathrm{e}^{-i \beta} \bar{B} \bar{C} V^{2} \\
-\frac{\bar{C}^{2} V^{2}}{r}+\frac{2 \lambda+\cos \theta}{r \sin \theta} \bar{A} \bar{C} U V
\end{array}\right] .
\end{gathered}
$$

\subsection{Calculation of 16 Christoffel Symbols}

We finally have all the pieces to finish the calculation of the Christoffel symbols. We encountered in (21) to (26) left and right terms:

$$
\begin{aligned}
\mathcal{L} & :=\frac{\nabla\left[\hat{\phi}\left(\sigma_{1}+i \sigma_{2}\right) \phi^{\dagger}\right]}{\operatorname{det}\left(\phi^{\dagger}\right)}+2 i q A \frac{\hat{\phi}\left(\sigma_{1}+i \sigma_{2}\right) \phi^{\dagger}}{\operatorname{det}\left(\phi^{\dagger}\right)} \\
& =\mathcal{S}_{(1)}+i \mathcal{S}_{(1)}^{\prime}+i\left(\mathcal{S}_{(2)}+i \mathcal{S}_{(2)}^{\prime}\right)+2 i q\left[\mathcal{A}_{(1)}+i \mathcal{A}_{(1)}^{\prime}+i\left(\mathcal{A}_{(2)}+i \mathcal{A}_{(2)}^{\prime}\right)\right] \\
& =\mathcal{S}_{(1)}-2 q \mathcal{A}_{(2)}-\left(\mathcal{S}_{(2)}^{\prime}+2 q \mathcal{A}_{(1)}^{\prime}\right)+i\left[\left(\mathcal{S}_{(1)}^{\prime}-2 q \mathcal{A}_{(2)}^{\prime}\right)+\left(\mathcal{S}_{(2)}+2 q \mathcal{A}_{(1)}\right)\right] .
\end{aligned}
$$

This allows us to calculate the four $\Gamma_{1 \mu}^{0}+\Gamma_{1 \mu}^{3}+i\left(\Gamma_{2 \mu}^{0}-\Gamma_{3 \mu}^{2}\right)$. Similarly:

$$
\begin{aligned}
\mathcal{R} & :=\frac{\nabla\left[\hat{\phi}\left(\sigma_{1}-i \sigma_{2}\right) \phi^{\dagger}\right]}{\operatorname{det}\left(\phi^{\dagger}\right)}-2 i q A \frac{\hat{\phi}\left(\sigma_{1}-i \sigma_{2}\right) \phi^{\dagger}}{\operatorname{det}\left(\phi^{\dagger}\right)} \\
& =\mathcal{S}_{(1)}+i \mathcal{S}_{(1)}^{\prime}-i\left(\mathcal{S}_{(2)}+i \mathcal{S}_{(2)}^{\prime}\right)-2 i q\left[\mathcal{A}_{(1)}+i \mathcal{A}_{(1)}^{\prime}-i\left(\mathcal{A}_{(2)}+i \mathcal{A}_{(2)}^{\prime}\right)\right]
\end{aligned}
$$




$$
=\mathcal{S}_{(1)}-2 q \mathcal{A}_{(2)}+\left(\mathcal{S}_{(2)}^{\prime}+2 q \mathcal{A}_{(1)}^{\prime}\right)+i\left[\left(\mathcal{S}_{(1)}^{\prime}-2 q \mathcal{A}_{(2)}^{\prime}\right)-\left(\mathcal{S}_{(2)}+2 q \mathcal{A}_{(1)}\right)\right]
$$

This allows to calculate the four $\Gamma_{1 \mu}^{0}-\Gamma_{1 \mu}^{3}-i\left(\Gamma_{2 \mu}^{0}+\Gamma_{3 \mu}^{2}\right)$. We let:

$$
\begin{gathered}
\mathcal{L}=: S\left(\mathbf{L}_{1}+i \mathbf{L}_{2}\right) S^{\dagger} ; \mathbf{L}_{1}=\mathbf{L}_{1}^{\dagger} ; \mathbf{L}_{2}=\mathbf{L}_{2}^{\dagger}, \\
\mathcal{R}=: S\left(\mathbf{R}_{1}+i \mathbf{R}_{2}\right) S^{\dagger} ; \mathbf{R}_{1}=\mathbf{R}_{1}^{\dagger} ; \mathbf{R}_{2}=\mathbf{R}_{2}^{\dagger},
\end{gathered}
$$

We obtain:

$$
\begin{aligned}
& \frac{\nabla\left[\hat{\phi}\left(\sigma_{1}+i \sigma_{2}\right) \phi^{\dagger}\right]}{\operatorname{det}\left(\phi^{\dagger}\right)}+2 i q A \frac{\hat{\phi}\left(\sigma_{1}+i \sigma_{2}\right) \phi^{\dagger}}{\operatorname{det}\left(\phi^{\dagger}\right)} \\
= & \mathcal{S}_{(1)}-2 q \mathcal{A}_{(2)}+i\left(\mathcal{S}_{(1)}^{\prime}-2 q \mathcal{A}_{(2)}^{\prime}\right)+i\left[\mathcal{S}_{(2)}+2 q \mathcal{A}_{(1)}+i\left(\mathcal{S}_{(2)}^{\prime}+2 q \mathcal{A}_{(1)}^{\prime}\right)\right] \\
& \Gamma_{1 \mu}^{0}-i \Gamma_{3 \mu}^{2}+i\left(\Gamma_{2 \mu}^{0}-i \Gamma_{1 \mu}^{3}\right)=\mathrm{d}_{\mu} \cdot \mathbf{L}_{1}+i \mathrm{~d}_{\mu} \cdot \mathbf{L}_{2}+2 m \rho\left(\delta_{\mu}^{2}-i \delta_{\mu}^{1}\right) .
\end{aligned}
$$

The separation between real and imaginary parts of this equality gives:

$$
\begin{gathered}
\Gamma_{1 \mu}^{0}+\Gamma_{1 \mu}^{3}=\mathrm{d}_{\mu} \cdot \mathbf{L}_{1}+2 m \rho \delta_{\mu}^{2}=\mathrm{d}_{\mu}^{0} \mathbf{L}_{1}^{0}-\mathrm{d}_{\mu}^{1} \mathbf{L}_{1}^{1}-\mathrm{d}_{\mu}^{2} \mathbf{L}_{1}^{2}-\mathrm{d}_{\mu}^{3} \mathbf{L}_{1}^{3}+2 m \rho \delta_{\mu}^{2}, \\
-\Gamma_{3 \mu}^{2}+\Gamma_{2 \mu}^{0}=\mathrm{d}_{\mu} \cdot \mathbf{L}_{2}-2 m \rho \delta_{\mu}^{1}=\mathrm{d}_{\mu}^{0} \mathbf{L}_{2}^{0}-\mathrm{d}_{\mu}^{1} \mathbf{L}_{2}^{1}-\mathrm{d}_{\mu}^{2} \mathbf{L}_{2}^{2}-\mathrm{d}_{\mu}^{3} \mathbf{L}_{2}^{3}-2 m \rho \delta_{\mu}^{1},
\end{gathered}
$$

Now we need:

$$
\begin{aligned}
& \frac{1}{2}\left(\mathcal{A}^{\prime}+\mathcal{D}^{\prime}\right)=\mathbf{L}_{1}^{0}+i \mathbf{L}_{2}^{0}=2 i m U V \mathrm{e}^{2 i \zeta} \frac{A B-C D}{\left|\operatorname{det}\left(X^{\dagger}\right)\right|} \\
& \frac{1}{2}\left(\mathcal{A}^{\prime}-\mathcal{D}^{\prime}\right)=\mathbf{L}_{1}^{3}+i \mathbf{L}_{2}^{3}=2 U V \mathrm{e}^{2 i \zeta} \frac{2 i\left(E+\frac{\alpha}{r}\right) D B+\frac{\kappa}{r}\left(B^{2}-D^{2}\right)}{\operatorname{det}\left(X^{\dagger}\right)}, \\
& \frac{1}{2}\left(\mathcal{B}^{\prime}+\mathcal{C}^{\prime}\right)=\mathbf{L}_{1}^{1}+i \mathbf{L}_{2}^{1} \\
& =2 i m e^{2 i \zeta} \frac{B C V^{2}-A D U^{2}}{\left|\operatorname{det}\left(X^{\dagger}\right)\right|} \\
& +\mathrm{e}^{2 i \zeta} \frac{B^{2} V^{2}+D^{2} U^{2}+2 \kappa\left(U^{2}+V^{2}\right) B D-\frac{4 \lambda}{\sin \theta} B D U V}{r \operatorname{det}\left(X^{\dagger}\right)}, \\
& -\frac{1}{2}\left(\mathcal{B}^{\prime}-\mathcal{C}^{\prime}\right)=-\mathbf{L}_{2}^{2}+i \mathbf{L}_{1}^{2} \\
& =-2 \mathrm{e}^{2 i \zeta} \frac{i m\left(A D U^{2}+B C V^{2}\right)}{\left|\operatorname{det}\left(X^{\dagger}\right)\right|} \\
& +\frac{\mathrm{e}^{2 i \zeta}}{\operatorname{det}\left(X^{\dagger}\right)}\left[\frac{1}{r}\left(D^{2} U^{2}-B^{2} V^{2}\right)+2 B D U V \frac{\cos \theta}{r \sin \theta}\right] .
\end{aligned}
$$

We let:

$$
\begin{aligned}
\rho_{0} \mathrm{e}^{i \delta_{0}}: & =\left(\mathbf{L}_{1}^{0}+i \mathbf{L}_{2}^{0}\right) \mathrm{e}^{-2 i \zeta} ; \rho_{3} \mathrm{e}^{i \delta_{3}}:=\left(\mathbf{L}_{1}^{3}+i \mathbf{L}_{2}^{3}\right) \mathrm{e}^{-2 i \zeta}, \\
\rho_{1} \mathrm{e}^{i \delta_{1}} & :=\left(\mathbf{L}_{1}^{1}+i \mathbf{L}_{2}^{1}\right) \mathrm{e}^{-2 i \zeta} ; \rho_{2} \mathrm{e}^{i \delta_{2}}:=\left(\mathbf{L}_{1}^{2}+i \mathbf{L}_{2}^{2}\right) \mathrm{e}^{-2 i \zeta} .
\end{aligned}
$$


This gives:

$$
\begin{aligned}
& \mathbf{L}_{1}^{0}=\rho_{0} \cos \left(2 \zeta+\delta_{0}\right) ; \mathbf{L}_{2}^{0}=\rho_{0} \sin \left(2 \zeta+\delta_{0}\right) \\
& \mathbf{L}_{1}^{1}=\rho_{1} \cos \left(2 \zeta+\delta_{1}\right) ; \mathbf{L}_{2}^{1}=\rho_{1} \sin \left(2 \zeta+\delta_{1}\right) \\
& \mathbf{L}_{1}^{2}=-\rho_{2} \cos \left(2 \zeta+\delta_{2}\right) ; \mathbf{L}_{2}^{2}=\rho_{2} \sin \left(2 \zeta+\delta_{2}\right) \\
& \mathbf{L}_{1}^{3}=\rho_{3} \cos \left(2 \zeta+\delta_{3}\right) ; \mathbf{L}_{2}^{3}=\rho_{3} \sin \left(2 \zeta+\delta_{3}\right) .
\end{aligned}
$$

Then (148) and (149) read:

$$
\begin{aligned}
\Gamma_{1 \mu}^{0}+\Gamma_{1 \mu}^{3}= & \mathrm{d}_{\mu}^{0} \rho_{0} \cos \left(2 \zeta+\delta_{0}\right)-\mathrm{d}_{\mu}^{1} \rho_{1} \cos \left(2 \zeta+\delta_{1}\right) \\
& +\mathrm{d}_{\mu}^{2} \rho_{2} \cos \left(2 \zeta+\delta_{2}\right)-\mathrm{d}_{\mu}^{3} \rho_{3} \cos \left(2 \zeta+\delta_{3}\right)+2 m \rho \delta_{\mu}^{2}, \\
\Gamma_{2 \mu}^{0}-\Gamma_{3 \mu}^{2}= & \mathrm{d}_{\mu}^{0} \rho_{0} \sin \left(2 \zeta+\delta_{0}\right)-\mathrm{d}_{\mu}^{1} \rho_{1} \sin \left(2 \zeta+\delta_{1}\right) \\
& -\mathrm{d}_{\mu}^{2} \rho_{2} \sin \left(2 \zeta+\delta_{2}\right)-\mathrm{d}_{\mu}^{3} \rho_{3} \sin \left(2 \zeta+\delta_{3}\right)-2 m \rho \delta_{\mu}^{1},
\end{aligned}
$$

These equations give for instance:

$$
\begin{aligned}
& \Gamma_{10}^{0}+\Gamma_{10}^{3}=\mathrm{d}_{0}^{0} \rho_{0} \cos \left(2 \zeta+\delta_{0}\right)-\mathrm{d}_{0}^{1} \rho_{1} \cos \left(2 \zeta+\delta_{1}\right) \\
& +\mathrm{d}_{0}^{2} \rho_{2} \cos \left(2 \zeta+\delta_{2}\right)-\mathrm{d}_{0}^{3} \rho_{3} \cos \left(2 \zeta+\delta_{3}\right) \text {, } \\
& \Gamma_{11}^{0}+i \Gamma_{12}^{0}+\Gamma_{11}^{3}+i \Gamma_{12}^{3} \\
& =\left(\mathrm{d}_{1}^{0}+i \mathrm{~d}_{2}^{0}\right) \rho_{0} \cos \left(2 \zeta+\delta_{0}\right)-\left(\mathrm{d}_{1}^{1}+i \mathrm{~d}_{2}^{1}\right) \rho_{1} \cos \left(2 \zeta+\delta_{1}\right) \\
& +\left(\mathrm{d}_{1}^{2}+i \mathrm{~d}_{2}^{2}\right) \rho_{2} \cos \left(2 \zeta+\delta_{2}\right)-\left(\mathrm{d}_{1}^{3}+i \mathrm{~d}_{2}^{3}\right) \rho_{3} \cos \left(2 \zeta+\delta_{3}\right)+2 i m \rho \\
& =\mathrm{e}^{2 i \zeta}\left(-R_{1} L_{2}+R_{2} L_{1}\right) \rho_{0} \cos \left(2 \zeta+\delta_{0}\right)-\mathrm{e}^{2 i \zeta}\left(R_{1} L_{1}-R_{2} L_{2}\right) \rho_{1} \cos \left(2 \zeta+\delta_{1}\right) \\
& +\mathrm{e}^{2 i \zeta}\left(i R_{1} L_{1}+i R_{2} L_{2}\right) \rho_{2} \cos \left(2 \zeta+\delta_{2}\right) \\
& -\mathrm{e}^{2 i \zeta}\left(-R_{1} L_{1}-R_{2} L_{2}\right) \rho_{3} \cos \left(2 \zeta+\delta_{3}\right)+2 i m \rho \text {. }
\end{aligned}
$$

The same calculation must be made for the right terms:

$$
\begin{aligned}
& \frac{\nabla\left[\hat{\phi}\left(\sigma_{1}-i \sigma_{2}\right) \phi^{\dagger}\right]}{\operatorname{det}\left(\phi^{\dagger}\right)}-2 i q A \frac{\hat{\phi}\left(\sigma_{1}-i \sigma_{2}\right) \phi^{\dagger}}{\operatorname{det}\left(\phi^{\dagger}\right)} \\
= & \mathcal{S}_{(1)}-2 q \mathcal{A}_{(2)}+i\left(\mathcal{S}_{(1)}^{\prime}-2 q \mathcal{A}_{(2)}^{\prime}\right)-i\left[\mathcal{S}_{(2)}+2 q \mathcal{A}_{(1)}+i\left(\mathcal{S}_{(2)}^{\prime}+2 q \mathcal{A}_{(1)}^{\prime}\right)\right] \\
& \Gamma_{1 \mu}^{0}-i \Gamma_{3 \mu}^{2}-i\left(\Gamma_{2 \mu}^{0}-i \Gamma_{1 \mu}^{3}\right)=\mathrm{d}_{\mu} \cdot \mathbf{R}_{1}+i \mathrm{~d}_{\mu} \cdot \mathbf{R}_{2}+2 m \rho\left(\delta_{\mu}^{2}+i \delta_{\mu}^{1}\right) .
\end{aligned}
$$

The separation between real and imaginary parts of this equality gives:

$$
\begin{gathered}
\Gamma_{1 \mu}^{0}-\Gamma_{1 \mu}^{3}=\mathrm{d}_{\mu} \cdot \mathbf{R}_{1}+2 m \rho \delta_{\mu}^{2}=\mathrm{d}_{\mu}^{0} \mathbf{R}_{1}^{0}-\mathrm{d}_{\mu}^{1} \mathbf{R}_{1}^{1}-\mathrm{d}_{\mu}^{2} \mathbf{R}_{1}^{2}-\mathrm{d}_{\mu}^{3} \mathbf{R}_{1}^{3}+2 m \rho \delta_{\mu}^{2}, \\
-\Gamma_{3 \mu}^{2}-\Gamma_{2 \mu}^{0}=\mathrm{d}_{\mu} \cdot \mathbf{R}_{2}+2 m \rho \delta_{\mu}^{1}=\mathrm{d}_{\mu}^{0} \mathbf{R}_{2}^{0}-\mathrm{d}_{\mu}^{1} \mathbf{R}_{2}^{1}-\mathrm{d}_{\mu}^{2} \mathbf{R}_{2}^{2}-\mathrm{d}_{\mu}^{3} \mathbf{R}_{2}^{3}+2 m \rho \delta_{\mu}^{1},
\end{gathered}
$$

Now we need (with $\rho_{X}=\left|\operatorname{det}\left(X^{\dagger}\right)\right|$ ):

$$
\begin{gathered}
\frac{1}{2}\left(\mathcal{A}_{-}^{\prime}+\mathcal{D}_{-}^{\prime}\right)=\mathbf{R}_{1}^{0}+i \mathbf{R}_{2}^{0}=2 i m U V \mathrm{e}^{-2 i \zeta} \frac{\bar{D} \bar{C}-\bar{A} \bar{B}}{\rho_{X}} \\
\frac{1}{2}\left(\mathcal{A}_{-}^{\prime}-\mathcal{D}_{-}^{\prime}\right)=\mathbf{R}_{1}^{3}+i \mathbf{R}_{2}^{3}=2 U V \mathrm{e}^{-2 i \zeta} \frac{-2 i\left(E+\frac{\alpha}{r}\right) \bar{A} \bar{C}+\frac{\kappa}{r}\left(\bar{A}^{2}-\bar{C}^{2}\right)}{\operatorname{det}\left(X^{\dagger}\right)},
\end{gathered}
$$




$$
\begin{aligned}
\frac{1}{2}\left(\mathcal{B}_{-}^{\prime}+\mathcal{C}_{-}^{\prime}\right)= & \mathbf{R}_{1}^{1}+i \mathbf{R}_{2}^{1} \\
= & 2 i m \mathrm{e}^{-2 i \zeta} \frac{\bar{D} \bar{A} U^{2}+\bar{B} \bar{C} V^{2}}{\rho_{X}} \\
& +\mathrm{e}^{-2 i \zeta} \frac{-\left(\bar{C}^{2} V^{2}+\bar{A}^{2} U^{2}\right)+2 \kappa\left(U^{2}+V^{2}\right) \bar{A} \bar{C}+\frac{4 \lambda}{\sin \theta} \bar{A} \bar{C} U V}{r \operatorname{det}\left(X^{\dagger}\right)}, \\
-\frac{1}{2}\left(\mathcal{B}_{-}^{\prime}-\mathcal{C}_{-}^{\prime}\right)= & -\mathbf{R}_{2}^{2}+i \mathbf{R}_{1}^{2} \\
= & \frac{\mathrm{e}^{-2 i \zeta}}{\operatorname{det}\left(X^{\dagger}\right)}\left[\frac{1}{r}\left(\bar{A}^{2} U^{2}-\bar{C}^{2} V^{2}\right)+2 \bar{A} \bar{C} U V \frac{\cos \theta}{r \sin \theta}\right] \\
& +2 \mathrm{e}^{-2 i \zeta} i m \frac{\bar{B} \bar{C} V^{2}-\bar{A} \bar{D} U^{2}}{\rho_{X}} .
\end{aligned}
$$

We let:

$$
\begin{aligned}
\rho_{0}^{\prime} \mathrm{e}^{i \delta_{0}^{\prime}}:=\mathrm{e}^{2 i \zeta}\left(\mathbf{R}_{1}^{0}+i \mathbf{R}_{2}^{0}\right) ; \rho_{3}^{\prime} \mathrm{e}^{i \delta_{3}^{\prime}}:=\mathrm{e}^{2 i \zeta}\left(\mathbf{R}_{1}^{3}+i \mathbf{R}_{2}^{3}\right), \\
\rho_{1}^{\prime} \mathrm{e}^{i \delta_{1}^{\prime}}:=\mathrm{e}^{2 i \zeta}\left(\mathbf{R}_{1}^{1}+i \mathbf{R}_{2}^{1}\right) ; \rho_{2}^{\prime} \mathrm{e}^{i \delta_{2}^{\prime}}:=\mathrm{e}^{2 i \zeta}\left(\mathbf{R}_{1}^{1}+i \mathbf{R}_{2}^{1}\right) .
\end{aligned}
$$

This gives:

$$
\begin{gathered}
\mathbf{R}_{1}^{0}=\rho_{0}^{\prime} \cos \left(-2 \zeta+\delta_{0}^{\prime}\right) ; \mathbf{R}_{2}^{0}=\rho_{0}^{\prime} \sin \left(-2 \zeta+\delta_{0}^{\prime}\right) \\
\mathbf{R}_{1}^{1}=\rho_{1}^{\prime} \cos \left(-2 \zeta+\delta_{1}^{\prime}\right) ; \mathbf{R}_{2}^{1}=\rho_{1}^{\prime} \sin \left(-2 \zeta+\delta_{1}^{\prime}\right) \\
\mathbf{R}_{1}^{2}=-\rho_{2}^{\prime} \cos \left(-2 \zeta+\delta_{2}^{\prime}\right) ; \mathbf{R}_{2}^{2}=\rho_{2}^{\prime} \sin \left(-2 \zeta+\delta_{2}^{\prime}\right) \\
\mathbf{R}_{1}^{3}=\rho_{3}^{\prime} \cos \left(-2 \zeta+\delta_{3}^{\prime}\right) ; \mathbf{R}_{2}^{3}=\rho_{3}^{\prime} \sin \left(-2 \zeta+\delta_{3}^{\prime}\right)
\end{gathered}
$$

Then (166) and (167) read:

$$
\begin{aligned}
\Gamma_{1 \mu}^{0}-\Gamma_{1 \mu}^{3}= & \mathrm{d}_{\mu}^{0} \rho_{0}^{\prime} \cos \left(-2 \zeta+\delta_{0}^{\prime}\right)-\mathrm{d}_{\mu}^{1} \rho_{1}^{\prime} \cos \left(-2 \zeta+\delta_{1}^{\prime}\right) \\
& +\mathrm{d}_{\mu}^{2} \rho_{2}^{\prime} \cos \left(-2 \zeta+\delta_{2}^{\prime}\right)-\mathrm{d}_{\mu}^{3} \rho_{3}^{\prime} \cos \left(-2 \zeta+\delta_{3}^{\prime}\right)+2 m \rho \delta_{\mu}^{2}, \\
-\Gamma_{2 \mu}^{0}-\Gamma_{3 \mu}^{2}= & \mathrm{d}_{\mu}^{0} \rho_{0}^{\prime} \sin \left(-2 \zeta+\delta_{0}^{\prime}\right)-\mathrm{d}_{\mu}^{1} \rho_{1}^{\prime} \sin \left(-2 \zeta+\delta_{1}^{\prime}\right) \\
& -\mathrm{d}_{\mu}^{2} \rho_{2}^{\prime} \sin \left(-2 \zeta+\delta_{2}^{\prime}\right)-\mathrm{d}_{\mu}^{3} \rho_{3}^{\prime} \sin \left(-2 \zeta+\delta_{3}^{\prime}\right)-2 m \rho \delta_{\mu}^{1},
\end{aligned}
$$

\section{Torsion and Symmetric Part of the Connection}

The 64 Christoffel symbols may be calculated from the $4 \times 7=28$ independent terms, using the 36 relations described in (16), (17) and (18):

$$
\begin{gathered}
\Gamma_{0 \mu}^{0}=\mathrm{d}_{\mu} \cdot \mathbf{K}_{0} ; \Gamma_{3 \mu}^{0}=\mathrm{d}_{\mu} \cdot \mathbf{K}_{3} ; \Gamma_{2 \mu}^{1}=-\mathrm{d}_{\mu} \cdot \mathbf{K}_{3}^{\prime}-2 m \rho \delta_{\mu}^{0} \\
\Gamma_{1 \mu}^{0}=\mathrm{d}_{\mu} \cdot \frac{\mathbf{L}_{1}+\mathbf{R}_{1}}{2}+2 m \rho \delta_{\mu}^{2} ; \Gamma_{1 \mu}^{3}=\mathrm{d}_{\mu} \cdot \frac{\mathbf{L}_{1}-\mathbf{R}_{1}}{2} \\
\Gamma_{2 \mu}^{0}=\mathrm{d}_{\mu} \cdot \frac{\mathbf{L}_{2}-\mathbf{R}_{2}}{2}-2 m \rho \delta_{\mu}^{1} ; \Gamma_{3 \mu}^{2}=\mathrm{d}_{\mu} \cdot \frac{-\mathbf{L}_{2}-\mathbf{R}_{2}}{2} .
\end{gathered}
$$

The torsion tensor is usually defined as:

$$
\mathbf{T}_{j k}^{\mu}:=\frac{1}{2}\left(\Gamma_{j k}^{\mu}-\Gamma_{k j}^{\mu}\right) ; j k=01,02,03,12,23,31, \mu=0,1,2,3 .
$$


This tensor is then antisymmetric: $\left(\mathbf{T}_{k j}^{\mu}:=-\mathbf{T}_{j k}^{\mu}\right)$. Here, for instance, eight terms contain the $\mathbf{K}$ space-time vectors, without other terms:

$$
\begin{aligned}
& \mathbf{T}_{30}^{0}=-\mathbf{T}_{03}^{0}=\frac{1}{2}\left[-\mathrm{d}_{3} \cdot \mathbf{K}_{0}+\mathrm{d}_{0} \cdot \mathbf{K}_{3}\right], \\
& \mathbf{T}_{12}^{1}=-\mathbf{T}_{21}^{1}=\frac{1}{2}\left[\mathrm{~d}_{1} \cdot \mathbf{K}_{3}^{\prime}+\mathrm{d}_{2} \cdot \mathbf{K}_{0}\right], \\
& \mathbf{T}_{12}^{2}=-\mathbf{T}_{21}^{2}=\frac{1}{2}\left[\mathrm{~d}_{2} \cdot \mathbf{K}_{3}^{\prime}-\mathrm{d}_{1} \cdot \mathbf{K}_{0}\right], \\
& \mathbf{T}_{30}^{3}=-\mathbf{T}_{03}^{3}=\frac{1}{2}\left[\mathrm{~d}_{0} \cdot \mathbf{K}_{0}-\mathrm{d}_{3} \cdot \mathbf{K}_{3}\right],
\end{aligned}
$$

Rodichev [55] studied the torsion in the frame of a Euclidean geometry with torsion. The present study acts in the frame of a space-time manifold, not Euclidean, so we get very different properties. In space-time the torsion tensor has 24 independent components while the connection contains 28 independent Christoffel symbols. We obtain these 28 symbols from the $4 \times 8=32$ functions $a_{\mu}^{n}$ defining a dilator $M$ (see ([41] 4.1.2), satisfying:

$$
M=1+\mathrm{dx}^{\mu}\left(a_{\mu}^{0}+a_{\mu}^{j} \sigma_{j}+a_{\mu}^{3+j} i \sigma_{j}+a_{\mu}^{7} i\right),
$$

where we have:

$$
\begin{gathered}
\Gamma_{0 \mu}^{0}=\Gamma_{1 \mu}^{1}=\Gamma_{2 \mu}^{2}=\Gamma_{3 \mu}^{3}=2 a_{\mu}^{0}, \\
\Gamma_{0 \mu}^{1}=\Gamma_{1 \mu}^{0}=2 a_{\mu}^{1} ; \Gamma_{0 \mu}^{2}=\Gamma_{2 \mu}^{0}=2 a_{\mu}^{2} ; \Gamma_{0 \mu}^{3}=\Gamma_{3 \mu}^{0}=2 a_{\mu}^{3}, \\
\Gamma_{3 \mu}^{2}=-\Gamma_{2 \mu}^{3}=2 a_{\mu}^{4} ; \Gamma_{1 \mu}^{3}=-\Gamma_{3 \mu}^{1}=2 a_{\mu}^{5} ; \Gamma_{2 \mu}^{1}=-\Gamma_{1 \mu}^{2}=2 a_{\mu}^{6} .
\end{gathered}
$$

\section{Concluding Remarks}

The principle of equivalence between inertia and gravitation being simply the equality between the $\Gamma_{\mu v}^{\rho}$ and the $\Gamma_{\mu v}^{\rho}$, the solutions studied here show that all 28 functions come simply from scalar products of the four contravariant $D_{\mu}$ by the seven covariant vectors:

$$
\begin{gathered}
\mathbf{V}_{0}:=S \mathbf{K}_{0} S^{\dagger} ; \mathbf{V}_{3}:=S \mathbf{K}_{3} S^{\dagger} ; \mathbf{V}_{6}:=S \mathbf{K}_{3}^{\prime} S^{\dagger}, \\
2 \mathcal{L}_{1}:=S \mathbf{L}_{1} S^{\dagger} ; 2 \mathcal{R}_{1}:=S \mathbf{R}_{1} S^{\dagger} ; 2 \mathcal{L}_{2}:=S \mathbf{L}_{2} S^{\dagger} ; 2 \mathcal{R}_{2}:=S \mathbf{R}_{2} S^{\dagger} .
\end{gathered}
$$

These vectors allow us to obtain all Christoffel symbols as scalar products:

$$
\begin{gathered}
\Gamma_{0 \mu}^{0}=\Gamma_{1 \mu}^{1}=\Gamma_{2 \mu}^{2}=\Gamma_{3 \mu}^{3}=2 a_{\mu}^{0}=D_{\mu} \cdot \mathbf{V}_{0}, \\
\Gamma_{0 \mu}^{3}=\Gamma_{3 \mu}^{0}=2 a_{\mu}^{3}=D_{\mu} \cdot \mathbf{V}_{3}, \\
\Gamma_{2 \mu}^{1}=-\Gamma_{1 \mu}^{2}=2 a_{\mu}^{6}=D_{\mu} \cdot \mathbf{V}_{6}-2 m \rho \delta_{\mu}^{0},
\end{gathered}
$$

while the 32 other symbols use the right and left vectors:

$$
\begin{gathered}
\Gamma_{0 \mu}^{1}=\Gamma_{1 \mu}^{0}=2 a_{\mu}^{1}=D_{\mu} \cdot\left(\mathcal{L}_{1}+\mathcal{R}_{1}\right)+2 m \rho \delta_{\mu}^{2}, \\
\Gamma_{0 \mu}^{2}=\Gamma_{2 \mu}^{0}=2 a_{\mu}^{2}=D_{\mu} \cdot\left(\mathcal{L}_{2}-\mathcal{R}_{2}\right)-2 m \rho \delta_{\mu}^{1},
\end{gathered}
$$




$$
\begin{gathered}
\Gamma_{3 \mu}^{2}=-\Gamma_{2 \mu}^{3}=2 a_{\mu}^{4}=D_{\mu} \cdot\left(-\mathcal{L}_{2}-\mathcal{R}_{2}\right), \\
\Gamma_{1 \mu}^{3}=-\Gamma_{3 \mu}^{1}=2 a_{\mu}^{5}=D_{\mu} \cdot\left(\mathcal{L}_{1}-\mathcal{R}_{1}\right) .
\end{gathered}
$$

The chiral structure of the connection appears here, from the fact that three definitions only act with indexes 0 and 3 while four definitions act with the left and right vectors. Moreover only the symbols containing the three indexes 1, 2, 0 , contain the mass term $2 m \rho$. This chirality is also linked to the electric gauge transformation, which acts everywhere in quantum mechanics, even in the non-relativistic case. It induces a rotation in the 1-2 plane, in the direction from 1 to 2 : the rotation transforming 1 into 2 transforms 2 into -1 . This partially remains in non-relativistic quantum mechanics, where the conservation of the probability density still acts.

The previous calculation must be completed by the examination of the different cases corresponding to the different quantum numbers characterizing the electron states. This will be carried out in the second part of this work. These states are different first from the sign of the $\kappa$ number. This number is present in the previous calculation, not only by the value of $\mathrm{E}$ which contains $\kappa^{2}$, but also directly in the $\frac{\kappa}{r}$ terms, and also in the $\lambda$ terms: the values of $\lambda$ are $-j,-j+1, \cdots, j-1, j$ with $j=|\kappa|-\frac{1}{2}$. The linking of the states with $\kappa>0$ to the states with $\kappa<0$ is not a one-to-one link: the other quantum numbers are the integer degree $n_{1}$ of angular polynomial functions and the integer degree $n_{2}$ of radial polynomial functions, and there is no state with $\kappa<0$ and $n_{2}=0$. Consequently $\mathbf{n}(\mathbf{n}+1)$ states exist with $\kappa>0$ and only $\mathbf{n}(\mathbf{n}-1)$ states with $\kappa<0$, for each principal quantum number $\mathbf{n}$. The symmetry between these two kinds of states is then a false symmetry. This mainly comes from the property demonstrated in the next appendix: even if Gegenbauer's polynomials are different in the cases $\kappa>0$ and $\kappa<0$, the angular functions $U$ and $V$ encountered in the previous calculation are exactly proportional. The result of this similarity is the very popular Pauli's tale of the spin-up, spin-down states which doubles the $\mathbf{n}^{2}$ number issued from the spectroscopy and also from the Schrödinger wave equation.

It is only a tale, even if $\mathbf{n}(\mathbf{n}+1)+\mathbf{n}(\mathbf{n}-1)=2 \mathbf{n}^{2}$, as may be seen in our previous calculation: the spin effect is much more complicated. The $\lambda$ factor (with half-integer value) present in the $\zeta=\lambda \varphi-E x^{0}+\delta$ electric phase of the electron wave is doubled in all the $2 \zeta$ terms, but is even quadrupled in the $\mathrm{e}^{2 i \zeta} \cos \left(2 \zeta+\delta_{n}\right)$ terms in (163). It then happens that space-time turns more rapidly than the wave (or the wave turns less rapidly than the space time). Space-time geometry defined by the Christoffel symbols is animated not only by waves with a $2 \zeta$ phase, but also by waves with a $4 \zeta$ phase. This kind of phase was first encountered in general relativity as waves with spin 2 . Our calculation shows that it is linked to the quantum wave of the electron, as suspected by Feynman [56]. 


\section{Conflicts of Interest}

The authors declare no conflicts of interest regarding the publication of this paper.

\section{References}

[1] de Broglie, L. (1924) Recherches sur la théorie des quantas.

[2] Dirac, P.A.M. (1928) Proceedings of the Royal Society of London, 117, 610-624. https://doi.org/10.1098/rspa.1928.0023

[3] de Broglie, L. (1934) L'électron magnétique. Hermann, Paris.

[4] Daviau, C. (1993) Equation de Dirac non linéaire. Ph.D. Thesis, Université de Nantes, Nantes.

[5] Daviau, C. (1996) Dirac Equation in the Clifford Algebra of Space. In: Clifford Algebras and Their Application in Mathematical Physics, Springer, Boston, 67-88. https://doi.org/10.1007/978-94-011-5036-1_8

[6] Daviau, C. (1997) Advances in Applied Clifford Algebras, 7, 175-194.

[7] Daviau, C. (1997) Annales de la Fondation Louis de Broglie, 22, 87-103.

[8] Daviau, C. (1998) Annales de la Fondation Louis de Broglie, 23, 27-37.

[9] Daviau, C. (1998) Annales de la Fondation Louis de Broglie, 26, 149-171.

[10] Daviau, C. (2004) Chiral Dirac Equations. In: Clifford Algebras: Applications to Mathematics, Physics, and Engineering, Birkhäuser, Boston, 431-450. https://doi.org/10.1007/978-1-4612-2044-2 28

[11] Daviau, C. (2005) Annales de la Fondation Louis de Broglie, 30, 409-428. https://aflb.minesparis.psl.eu/AFLB-303/aflb303m398.pdf

[12] Daviau, C. (2008) Annales de la Fondation Louis de Broglie, 33, 53-67. https://aflb.minesparis.psl.eu/AFLB-331/aflb331m626.pdf

[13] Daviau, C. (2009) Annales de la Fondation Louis de Broglie, 34, 45-65. https://aflb.minesparis.psl.eu/AFLB-341/aflb341m648.pdf

[14] Daviau, C. (2010) Annales de la Fondation Louis de Broglie, 35, 51-78. https://aflb.minesparis.psl.eu/AFLB-351/aflb351m687.htm

[15] Daviau, C. (2011) L'espace-temps double. JePublie, Pouillé-les-coteaux. http://www.jepublie.com/livre-1-espace-temps-double-622.aspx

[16] Daviau, C. (2012) Advances in Applied Clifford Algebras, 22, 611-623. https://doi.org/10.1007/s00006-012-0351-7

[17] Daviau, C. (2009) Annales de la Fondation Louis de Broglie, 37, 253-268. https://aflb.minesparis.psl.eu/AFLB-371/aflb371m744.pdf

[18] Daviau, C. (2012) Double Space-Time and More. JePublie, Pouillé-les-coteaux. http://www.jepublie.com/livre-double-space-time-800.aspx

[19] Daviau, C. (2012) Nonlinear Dirac Equation, Magnetic Monopoles and Double Space-Time. CISP, Cambridge. https://hal.archives-ouvertes.fr/hal-01058706/document

[20] Daviau, C. and Bertrand, J. (2013) Annales de la Fondation Louis de Broglie, 38, 57.

[21] Daviau, C. (2013) Advances in Imaging and Electron Physics, 179, 1-136. https://doi.org/10.1016/B978-0-12-407700-3.00001-6

[22] Daviau, C. (2014) Advances in Applied Clifford Algebras, 22, 611-623. https://doi.org/10.1007/s00006-012-0351-7 
[23] Daviau, C. and Bertrand, J. (2014) New Insights in the Standard Model of Quantum Physics in Clifford Algebra. Je Publie, Pouillé-les-coteaux.

https://doi.org/10.1142/9780

https://hal.archives-ouvertes.fr/hal-00907848

[24] Daviau, C. and Bertrand, J. (2014) Journal of Modern Physics, 5, 1001-1022. https://doi.org/10.4236/jmp.2014.511102

[25] Daviau, C. and Bertrand, J. (2014) Journal of Modern Physics, 5, 2149-2173. https://doi.org/10.4236/jmp.2014.518210

[26] Daviau, C. and Bertrand, J. (2014) Annales de la Fondation Louis de Broglie, 40, 181-209.

https://aflb.minesparis.psl.eu/AFLB-401/aflb401m842.pdf

[27] Daviau, C. (2015) Annales de la Fondation Louis de Broglie, 40, 113-138. https://aflb.minesparis.psl.eu/AFLB-401/aflb401m807.pdf

[28] Daviau, C. and Bertrand, J. (2015) Journal of Modern Physics, 6, 2080-2092. https://doi.org/10.4236/jmp.2015.614215

[29] Daviau, C. and Bertrand, J. (2015) Journal of Applied Mathematics and Physics, 3, 46-61. https://doi.org/10.4236/jamp.2015.31007

[30] Daviau, C. and Bertrand, J. (2015) Journal of Modern Physics, 6, 1647-1656. https://doi.org/10.4236/jmp.2015.611166

[31] Daviau, C. (2016) Annales de la Fondation Louis de Broglie, 41, 73-97. https://aflb.minesparis.psl.eu/AFLB-401/aflb401m807.pdf

[32] Daviau, C. and Bertrand, J. (2016) The Standard Model of Quantum Physics in Clifford Algebra. World Scientific, Singapore.

https://www.worldscientific.com/worldscibooks/10.1142/9780 https://doi.org/10.1142/9780

[33] Daviau, C. and Bertrand, J. (2016) Journal of Modern Physics, 7, 936-951. https://doi.org/10.4236/jmp.2016.79086

[34] Daviau, C., Bertrand, J. and Girardot, D. (2016) Journal of Modern Physics, 7, 1568-1590. https://doi.org/10.4236/jmp.2016.712143

[35] Daviau, C., Bertrand, J. and Girardot, D. (2016) Journal of Modern Physics, 7, 2398-2417. https://doi.org/10.4236/jmp.2016.716207

[36] Daviau, C., Bertrand, J., Girardot, D. and Socroun, T. (2017) Annales de la Fondation Louis de Broglie, 42, 351-378. https://aflb.minesparis.psl.eu/AFLB-422/aflb422m873.pdf

[37] Daviau, C. and Bertrand, J. (2016) Journal of Modern Physics, 9, 250-258. https://doi.org/10.4236/jmp.2018.92017

[38] Daviau, C. (2019) Annales de la Fondation Louis de Broglie, 44, 163-186. https://aflb.minesparis.psl.eu/AFLB-441/aflb441m920.pdf

[39] Daviau, C., Bertrand, J., Socroun, T. and Girardot, D. (2019) Modèle Standard et Gravitation. Presses des Mines, Paris.

https://www.pressesdesmines.com/produit/modele-standard-et-gravitation

[40] Daviau, C. (2020) Dialogue pour une nouvelle physique (secondeödition). Ed. St Honoré, Paris.

https://www.editions-saint-honore.com/produit/dialogue-pour-une-nouvelle-physi que

[41] Daviau, C., Bertrand, J., Socroun, T. and Girardot, D. (2020) Developing a Theory of Everything. Annales de la Fondation Louis de Broglie, Paris. https://aflb.minesparis.psl.eu/MEMOS/ToEAFLB.pdf 
[42] Daviau, C., Bertrand, J. and Ng, R.A. (2020) Journal of Modern Physics, 11, 1075-1090. https://doi.org/10.4236/jmp.2020.117068

[43] Daviau, C. and Bertrand, J. (2020) Journal of Modern Physics, 11, 1263-1278. https://doi.org/10.4236/jmp.2020.119079

[44] Hestenes, D. (2020) Journal of Mathematical Physics, 14, 893-905. https://doi.org/10.1063/1.1666413

[45] Hestenes, D. (1986) A Unified Language for Mathematics and Physics and Clifford Algebra and the Interpretation of Quantum Mechanics. Reidel, Dordrecht. https://doi.org/10.1007/978-94-009-4728-3 27

[46] Lochak, G. (1983) Annales de la Fondation Louis de Broglie, 8, 345.

[47] Lochak, G. (1984) Annales de la Fondation Louis de Broglie, 9, 5e30.

[48] Lochak, G. (1985) International Journal of Theoretical Physics, 24, 1019-1050. https://doi.org/10.1007/BF00670815

[49] Lochak, G. (1995) The Symmetry between Electricity and Magnetism and the Problem of the Existence of a Magnetic Monopole. In: Barrett, T.W. and Grimes, D.M., Eds., Advanced Electromagnetism, World Scientific, Singapore, 105-147. https://doi.org/10.1142/9789812831323 0004

[50] Lochak, G. (2004) Annales de la Fondation Louis de Broglie, 29, 297-316. https://aflb.minesparis.psl.eu/AFLB-291/aflb291p297.htm

[51] Lochak, G. (2006) Annales de la Fondation Louis de Broglie, 31, 193-206. https://aflb.minesparis.psl.eu/AFLB-312/aflb312m508.htm

[52] Lochak, G. (2007) Annales de la Fondation Louis de Broglie, 32, 125-136. https://aflb.minesparis.psl.eu/AFLB-322/aflb322m610.htm

[53] Lochak, G. (2010) Annales de la Fondation Louis de Broglie, 35, 1-18. https://aflb.minesparis.psl.eu/AFLB-351/aflb351m704.htm

[54] Krüger, H. (1991) New Solutions of the Dirac Equation for Central Fields. In: Hestenes, D. and Weingartshofer, A., Eds., The Electron, Kluwer, Dordrecht, 49-81. https://link.springer.com/chapter/10.1007/978-94-011-3570-2 4 https://doi.org/10.1007/978-94-011-3570-2 4

[55] Rodichev, V.I. (1961) Journal of Experimental and Theoretical Physics, 40, 1469-1472. http://www.jetp.ac.ru/cgi-bin/dn/e 01305 1029.pdf

[56] Feynman, R. (1995) Lectures on Gravitation. Addison-Wesley, Boston. 


\section{Appendix: Angular Functions}

The angular functions are calculated from the auxiliary $C(\theta)$ function using the differential equation of Gegenbauer's polynomials (see [41] C.2):

$$
C(\theta)=\sum_{n=0}^{\infty} \frac{\left(|\lambda|-\kappa-\frac{1}{2}\right)_{n}\left(|\lambda|+\kappa+\frac{1}{2}\right)_{n}}{\left(\frac{1}{2}+|\lambda|\right)_{n} n !} \sin ^{2 n}\left(\frac{\theta}{2}\right),
$$

where $\kappa$ is any integer not equal to zero. This condition on $\kappa$ results from the necessity of the normalization of the $\phi$ wave. We first consider the case $\kappa>0$ and $\lambda>0$ for which we must have $\lambda=\frac{1}{2}, \frac{3}{2}, \cdots, \kappa-\frac{1}{2}$ (for other values of $\lambda$ and if $\kappa=0$ the $\phi$ function is ill-defined):

$$
C(\theta)=\sum_{n=0}^{\infty} \frac{\left(\lambda-\kappa-\frac{1}{2}\right)_{n}\left(\lambda+\kappa+\frac{1}{2}\right)_{n}}{\left(\frac{1}{2}+\lambda\right)_{n} n !} \sin ^{2 n}\left(\frac{\theta}{2}\right),
$$

with:

$$
(a)_{0}:=1 ;(a)_{1}:=a ;(a)_{n+1}:=a(a+1) \cdots(a+n) .
$$

We then have:

$$
\left(\lambda-\kappa-\frac{1}{2}\right)_{-\lambda+\kappa+\frac{1}{2}+1}=\left(\lambda-\kappa-\frac{1}{2}\right) \cdots(-2)(-1) 0=0 .
$$

And any other term with upper $n$ is also null. Then the infinite sum is reduced to:

$$
C(\theta)=\sum_{n=0}^{n=\kappa-\lambda+\frac{1}{2}} \frac{\left(\lambda-\kappa-\frac{1}{2}\right)_{n}\left(\lambda+\kappa+\frac{1}{2}\right)_{n}}{\left(\frac{1}{2}+\lambda\right)_{n} n !} \sin ^{2 n}\left(\frac{\theta}{2}\right),
$$

whose derivative is:

$$
C^{\prime}(\theta)=\cos \left(\frac{\theta}{2}\right)^{n=\kappa-\lambda+\frac{1}{2}} \sum_{n=1}^{\left(\lambda-\kappa-\frac{1}{2}\right)_{n}\left(\lambda+\kappa+\frac{1}{2}\right)_{n}} n \sin ^{2 n-1}\left(\frac{\theta}{2}\right) .
$$

All angular functions satisfy:

$$
\begin{aligned}
& \frac{U}{\sin ^{\lambda}(\theta)}=\left[\sin \left(\frac{\theta}{2}\right) C^{\prime}-\left(\kappa-\lambda+\frac{1}{2}\right) \cos \left(\frac{\theta}{2}\right) C\right], \\
& \frac{V}{\sin ^{\lambda}(\theta)}=\left[\cos \left(\frac{\theta}{2}\right) C^{\prime}+\left(\kappa-\lambda+\frac{1}{2}\right) \sin \left(\frac{\theta}{2}\right) C\right] .
\end{aligned}
$$

Since the term of $C^{\prime}$ with rank 0 is null from the $n$ factor: 


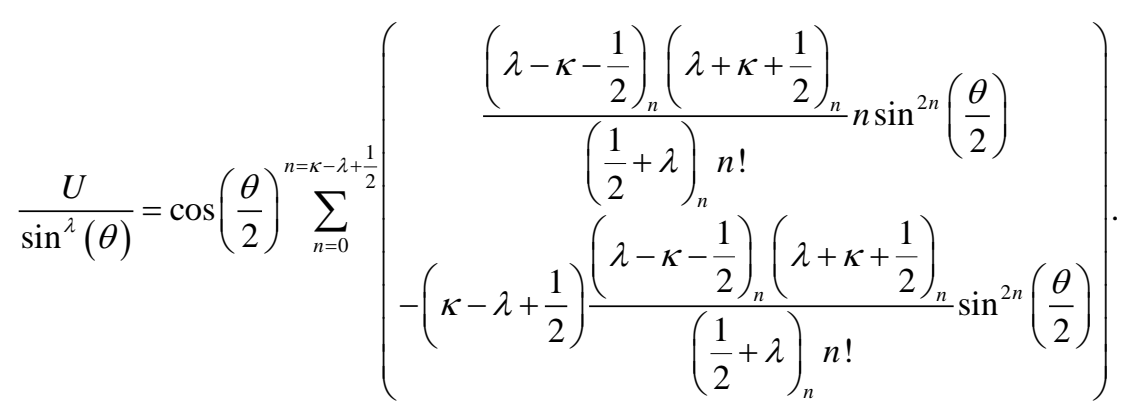

The last term of this sum is null, as it is a difference of two equal terms. Then the sum contains one term less and we obtain:

$\frac{U}{\sin ^{\lambda}(\theta)}=\cos \left(\frac{\theta}{2}\right)^{n=\kappa-\lambda-\frac{1}{2}} \sum_{n=0}\left[n-\left(\kappa-\lambda+\frac{1}{2}\right)\right] \frac{\left(\lambda-\kappa-\frac{1}{2}\right)_{n}\left(\lambda+\kappa+\frac{1}{2}\right)_{n}}{\left(\frac{1}{2}+\lambda\right)_{n} n !} \sin ^{2 n}\left(\frac{\theta}{2}\right)$.

And we have:

$$
\begin{aligned}
(a)_{n}(a+n) & =[a(a+1) \cdots(a+n-1)](a+n) \\
& =a[(a+1) \cdots(a+n-1)(a+n)]=a(a+1)_{n} .
\end{aligned}
$$

which implies:

$$
\left[n-\left(\kappa-\lambda+\frac{1}{2}\right)\right]\left(\lambda-\kappa-\frac{1}{2}\right)_{n}=\left(\lambda-\kappa-\frac{1}{2}\right)\left(\lambda-\kappa+\frac{1}{2}\right)_{n} .
$$

We then finally obtain:

$$
\frac{U}{\sin ^{\lambda}(\theta)}=\left(\lambda-\kappa-\frac{1}{2}\right) \cos \left(\frac{\theta}{2}\right) \sum_{n=0}^{n=\kappa-\lambda-\frac{1}{2}} \frac{\left(\lambda-\kappa+\frac{1}{2}\right)_{n}\left(\lambda+\kappa+\frac{1}{2}\right)_{n}}{\left(\frac{1}{2}+\lambda\right)_{n} n !} \sin ^{2 n}\left(\frac{\theta}{2}\right) .
$$

Next for $V$ we have:

$$
\begin{aligned}
\frac{V}{\sin ^{\lambda}(\theta)}= & {\left[1-\sin ^{2}\left(\frac{\theta}{2}\right)\right] \sum_{n=0}^{n=\kappa-\lambda+\frac{1}{2}} n \frac{\left(\lambda-\kappa-\frac{1}{2}\right)_{n}\left(\lambda+\kappa+\frac{1}{2}\right)_{n}}{\left(\frac{1}{2}+\lambda\right)_{n} n !} \sin ^{2 n-1}\left(\frac{\theta}{2}\right) } \\
& +\left(\kappa+\frac{1}{2}-\lambda\right) \sum_{n=0}^{n=\kappa-\lambda+\frac{1}{2}} \frac{\left(\lambda-\kappa-\frac{1}{2}\right)_{n}\left(\lambda+\kappa+\frac{1}{2}\right)_{n}}{\left(\frac{1}{2}+\lambda\right)_{n} n !} \sin ^{2 n+1}\left(\frac{\theta}{2}\right)
\end{aligned}
$$

Therefore we have:

$$
\begin{aligned}
\frac{V}{\sin ^{\lambda}(\theta)}= & \sum_{n=1}^{n=\kappa-\lambda+\frac{1}{2}} n \frac{\left(\lambda-\kappa-\frac{1}{2}\right)_{n}\left(\lambda+\kappa+\frac{1}{2}\right)_{n}}{\left(\frac{1}{2}+\lambda\right)_{n} n !} \sin ^{2 n-1}\left(\frac{\theta}{2}\right) \\
& +\left(-n+\kappa+\frac{1}{2}-\lambda\right)^{n=\kappa-\lambda+\frac{1}{2}} \frac{\left(\lambda-\kappa-\frac{1}{2}\right)_{n}\left(\lambda+\kappa+\frac{1}{2}\right)_{n}}{\left(\frac{1}{2}+\lambda\right)_{n} n !} \sin ^{2 n+1}\left(\frac{\theta}{2}\right)
\end{aligned}
$$


In the first sum we let $n=1+n^{\prime}$ while in the second sum we use the cancellation of the last term, so we have one term less:

$$
\begin{aligned}
\frac{V}{\sin ^{\lambda}(\theta)}= & \sum_{n^{\prime}=0}^{n^{\prime}=\kappa-\lambda-\frac{1}{2}} \frac{\left(\lambda-\kappa-\frac{1}{2}\right)_{1+n^{\prime}}\left(\lambda+\kappa+\frac{1}{2}\right)_{1+n^{\prime}}}{\left(\frac{1}{2}+\lambda\right)_{1+n^{\prime}} n^{\prime} !} \sin ^{2 n^{\prime}+1}\left(\frac{\theta}{2}\right) \\
& +\sum_{n=0}^{n=\kappa-\lambda-\frac{1}{2}} \frac{\left(\lambda-\kappa-\frac{1}{2}\right)_{n}\left(\lambda+\kappa+\frac{1}{2}\right)_{n}}{\left(\frac{1}{2}+\lambda\right)_{n} n !}\left(-n+\kappa+\frac{1}{2}-\lambda\right) \sin ^{2 n+1}\left(\frac{\theta}{2}\right) .
\end{aligned}
$$

In the first sum the $n^{\prime}$ variable is replaced by $n$. In the second sum the $\left(-n+\kappa+\frac{1}{2}-\lambda\right)$ factor gives also one term less and we obtain:

$$
\begin{aligned}
\frac{V}{\sin ^{\lambda}(\theta)}= & \sum_{n=0}^{n=\kappa-\lambda-\frac{1}{2}} \frac{\left(\lambda-\kappa-\frac{1}{2}\right)_{1+n}\left(\lambda+\kappa+\frac{1}{2}\right)_{1+n}}{\left(\frac{1}{2}+\lambda\right)_{1+n} n !} \sin ^{2 n+1}\left(\frac{\theta}{2}\right) \\
& +\sum_{n=0}^{n=\kappa-\lambda-\frac{1}{2}} \frac{\left(\lambda-\kappa-\frac{1}{2}\right)_{n}\left(\lambda+\kappa+\frac{1}{2}\right)_{n}}{\left(\frac{1}{2}+\lambda\right)_{n} n !}\left(-n+\kappa+\frac{1}{2}-\lambda\right) \sin ^{2 n+1}\left(\frac{\theta}{2}\right) .
\end{aligned}
$$

And we have:

$$
\begin{gathered}
\left(\lambda-\kappa-\frac{1}{2}\right)_{1+n}=\left(\lambda-\kappa-\frac{1}{2}\right)\left(\lambda-\kappa+\frac{1}{2}\right)_{n}, \\
\left(\lambda+\kappa+\frac{1}{2}\right)_{1+n}=\left(\lambda+\kappa+\frac{1}{2}\right)_{n}\left(\lambda+\kappa+\frac{1}{2}+n\right), \\
\left(\lambda-\kappa-\frac{1}{2}\right)_{n}\left(-n+\kappa+\frac{1}{2}-\lambda\right)=-\left(\lambda-\kappa-\frac{1}{2}\right)\left(\lambda-\kappa+\frac{1}{2}\right)_{n} .
\end{gathered}
$$

Then we obtain:

$$
\begin{aligned}
\frac{V}{\sin ^{\lambda}(\theta)}= & \left(\lambda-\kappa-\frac{1}{2}\right)^{n=\kappa-\lambda-\frac{1}{2}} \sum_{n=0} \frac{\left(\lambda-\kappa+\frac{1}{2}\right)_{n}\left(\lambda+\kappa+\frac{1}{2}\right)_{n}}{\left(\frac{1}{2}+\lambda\right)_{n} n !} \\
& \times\left[\frac{\lambda+\kappa+\frac{1}{2}+n}{\lambda+\frac{1}{2}+n}-1\right] \sin ^{2 n+1}\left(\frac{\theta}{2}\right) \\
= & \kappa\left(\lambda-\kappa-\frac{1}{2}\right)^{n=\kappa-\lambda-\frac{1}{2}} \frac{\left(\lambda-\kappa+\frac{1}{2}\right)_{n}\left(\lambda+\kappa+\frac{1}{2}\right)_{n}}{\left(\frac{1}{2}+\lambda\right)_{1+n} n !} \sin ^{2 n+1}\left(\frac{\theta}{2}\right) .
\end{aligned}
$$

Consider now the case $\kappa<0$ that means $\kappa=-|\kappa|$. Still for $\lambda>0$ we have 
now $\lambda=\frac{1}{2}, \frac{3}{2}, \cdots,|\kappa|-\frac{1}{2}$, and:

$$
C(\theta)=\sum_{n=0}^{\infty} \frac{\left(\lambda+|\kappa|-\frac{1}{2}\right)_{n}\left(\lambda-|\kappa|+\frac{1}{2}\right)_{n}}{\left(\frac{1}{2}+\lambda\right)_{n} n !} \sin ^{2 n}\left(\frac{\theta}{2}\right)
$$

Now it is the second factor which contains a negative integer and then this product cancels from some rank. Since this rank is (for a negative - $a$ integer) the a rank, we have:

$$
C(\theta)=\sum_{n=0}^{n=|\kappa|-\lambda-\frac{1}{2}} \frac{\left(\lambda+|\kappa|-\frac{1}{2}\right)_{n}\left(\lambda-|\kappa|+\frac{1}{2}\right)_{n}}{\left(\frac{1}{2}+\lambda\right)_{n} n !} \sin ^{2 n}\left(\frac{\theta}{2}\right),
$$

We see that this sum has one term less than in the case $\kappa>0$. We could expect that $U$ and $V$ should be very different from the functions previously calculated. Yet we will see that this is untrue. We have:

$$
C^{\prime}(\theta)=\cos \left(\frac{\theta}{2}\right) \sum_{n=0}^{n=|\kappa|-\lambda-\frac{1}{2}} n \frac{\left(\lambda+|\kappa|-\frac{1}{2}\right)_{n}\left(\lambda-|\kappa|+\frac{1}{2}\right)_{n}}{\left(\frac{1}{2}+\lambda\right)_{n} n !} \sin ^{2 n-1}\left(\frac{\theta}{2}\right)
$$

(206) gives for $U$ :

$$
\begin{aligned}
\frac{U}{\sin ^{\lambda}(\theta)}= & \sin \left(\frac{\theta}{2}\right) \cos \left(\frac{\theta}{2}\right) \sum_{n=0}^{n=|\kappa|-\lambda-\frac{1}{2}} n \frac{\left(\lambda+|\kappa|-\frac{1}{2}\right)_{n}\left(\lambda-|\kappa|+\frac{1}{2}\right)_{n}}{\left(\frac{1}{2}+\lambda\right)_{n} n !} \sin ^{2 n-1}\left(\frac{\theta}{2}\right) \\
& +\left(\lambda+|\kappa|-\frac{1}{2}\right) \cos \left(\frac{\theta}{2}\right) \sum_{n=0}^{n=|\kappa|-\lambda-\frac{1}{2}} \frac{\left(\lambda+|\kappa|-\frac{1}{2}\right)_{n}\left(\lambda-|\kappa|+\frac{1}{2}\right)_{n}}{\left(\frac{1}{2}+\lambda\right)_{n} n !} \sin ^{2 n}\left(\frac{\theta}{2}\right) .
\end{aligned}
$$

Then distributing the product in the second sum, we have:

$$
\begin{aligned}
\frac{U}{\sin ^{\lambda}(\theta)}= & \cos \left(\frac{\theta}{2}\right)^{n=|\kappa|-\lambda-\frac{1}{2}} \sum_{n=0}^{2}\left(n+\lambda+|\kappa|-\frac{1}{2}\right) \\
& \times \frac{\left(\lambda+|\kappa|-\frac{1}{2}\right)_{n}\left(\lambda-|\kappa|+\frac{1}{2}\right)_{n}}{\left(\frac{1}{2}+\lambda\right)_{n} n !} \sin ^{2 n}\left(\frac{\theta}{2}\right) .
\end{aligned}
$$

And we have with (211):

$$
\left(n+\lambda+|\kappa|-\frac{1}{2}\right)\left(\lambda+|\kappa|-\frac{1}{2}\right)_{n}=\left(\lambda+|\kappa|-\frac{1}{2}\right)\left(\lambda+|\kappa|+\frac{1}{2}\right)_{n} .
$$

We then obtain: 


$$
\frac{U}{\sin ^{\lambda}(\theta)}=\left(\lambda+|\kappa|-\frac{1}{2}\right) \cos \left(\frac{\theta}{2}\right) \sum_{n=0}^{n=|\kappa|-\lambda-\frac{1}{2}} \frac{\left(\lambda+|\kappa|+\frac{1}{2}\right)_{n}\left(\lambda-|\kappa|+\frac{1}{2}\right)_{n}}{\left(\frac{1}{2}+\lambda\right)_{n} n !} \sin ^{2 n}\left(\frac{\theta}{2}\right) .
$$

Comparison with (212) indicates, for $\lambda>0$ :

$$
U_{(\kappa=|\kappa|)}=\frac{\lambda+|\kappa|-\frac{1}{2}}{\lambda-|\kappa|-\frac{1}{2}} U_{(\kappa=-|\kappa|)} .
$$

Next for $V$ we start from (207) which gives:

$$
\begin{aligned}
\frac{V}{\sin ^{\lambda}(\theta)}= & \cos ^{2}\left(\frac{\theta}{2}\right) \sum_{n=0}^{n=|\kappa|-\lambda-\frac{1}{2}} n \frac{\left(\lambda+|\kappa|-\frac{1}{2}\right)_{n}\left(\lambda-|\kappa|+\frac{1}{2}\right)_{n}}{\left(\frac{1}{2}+\lambda\right)_{n} n !} \sin ^{2 n-1}\left(\frac{\theta}{2}\right) \\
& -\left(\lambda+|\kappa|-\frac{1}{2}\right)^{n=|\kappa|-\lambda-\frac{1}{2}} \sum_{n=0}^{\left(\lambda+|\kappa|-\frac{1}{2}\right)_{n}\left(\lambda-|\kappa|+\frac{1}{2}\right)_{n}} \sin ^{2 n+1}\left(\frac{\theta}{2}\right) .
\end{aligned}
$$

The first sum splits into two sums by using $\cos ^{2}(a)=1-\sin ^{2}(a)$ :

$$
\begin{aligned}
& \frac{V}{\sin ^{\lambda}(\theta)}=\sum_{n=0}^{n=|\kappa|-\lambda-\frac{1}{2}} n \frac{\left(\lambda+|\kappa|-\frac{1}{2}\right)_{n}\left(\lambda-|\kappa|+\frac{1}{2}\right)_{n}}{\left(\frac{1}{2}+\lambda\right)_{n} n !} \sin ^{2 n-1}\left(\frac{\theta}{2}\right) \\
& -\sum_{n=0}^{n=|\kappa|-\lambda-\frac{1}{2}} n \frac{\left(\lambda+|\kappa|-\frac{1}{2}\right)_{n}\left(\lambda-|\kappa|+\frac{1}{2}\right)_{n}}{\left(\frac{1}{2}+\lambda\right)_{n} n !} \sin ^{2 n+1}\left(\frac{\theta}{2}\right) \\
& -\left(\lambda+|\kappa|-\frac{1}{2}\right)^{n=|\kappa|-\lambda-\frac{1}{2}} \sum_{n=0} \frac{\left(\lambda+|\kappa|-\frac{1}{2}\right)_{n}\left(\lambda-|\kappa|+\frac{1}{2}\right)_{n}}{\left(\frac{1}{2}+\lambda\right)_{n} n !} \sin ^{2 n+1}\left(\frac{\theta}{2}\right) \text {. }
\end{aligned}
$$

The first sum begins truly with $n=1$ and we let $n=1+n^{\prime}$. We group together the last two sums:

$$
\begin{aligned}
\frac{V}{\sin ^{\lambda}(\theta)}= & \sum_{1+n^{\prime}=1}^{1+n^{\prime}=|\kappa|-\lambda-\frac{1}{2}} \frac{\left(\lambda+|\kappa|-\frac{1}{2}\right)_{1+n^{\prime}}\left(\lambda-|\kappa|+\frac{1}{2}\right)_{1+n^{\prime}}}{\left(\frac{1}{2}+\lambda\right)_{1+n^{\prime}} n^{\prime} !} \sin ^{2 n^{\prime}+1}\left(\frac{\theta}{2}\right) \\
& -\left(n+\lambda+|\kappa|-\frac{1}{2}\right)^{|\kappa|-\lambda-\frac{1}{2}\left(\lambda+|\kappa|-\frac{1}{2}\right)_{n}\left(\lambda-|\kappa|+\frac{1}{2}\right)_{n}} \sin _{n=0}^{2 n+1}\left(\frac{\theta}{2}\right) .
\end{aligned}
$$

In the first sum we replace $n^{\prime}$ by $n$ and we add a null term that changes 
nothing. In the second sum we again use (211), this gives:

$$
\begin{aligned}
\frac{V}{\sin ^{\lambda}(\theta)}= & \sum_{n=0}^{n=|\kappa|-\lambda-\frac{1}{2}} \frac{\left(\lambda+|\kappa|-\frac{1}{2}\right)_{1+n}\left(\lambda-|\kappa|+\frac{1}{2}\right)_{1+n}}{\left(\frac{1}{2}+\lambda\right)_{1+n} n !} \sin ^{2 n+1}\left(\frac{\theta}{2}\right) \\
& -\left(\lambda+|\kappa|-\frac{1}{2}\right)^{|\kappa|-\lambda-\frac{1}{2}\left(\lambda+|\kappa|+\frac{1}{2}\right)_{n}\left(\lambda-|\kappa|+\frac{1}{2}\right)_{n}} \sin _{n=0}^{2 n+1}\left(\frac{\theta}{2}\right) .
\end{aligned}
$$

This implies:

$$
\begin{aligned}
\frac{V}{\sin ^{\lambda}(\theta)}= & \left.\left(\lambda+|\kappa|-\frac{1}{2}\right)^{n=|\kappa|-\lambda-\frac{1}{2}\left(\lambda-|\kappa|+\frac{1}{2}\right)_{n}\left(\lambda+|\kappa|+\frac{1}{2}\right)_{n}} \frac{\left(\frac{1}{2}+\lambda\right)_{n} n !}{\left(\lambda+\frac{1}{2}+n\right.}\right]-1 \sin ^{2 n+1}\left(\frac{\theta}{2}\right) \\
& \times\left[\frac{\lambda-|\kappa|+\frac{1}{2}+n}{\left(\frac{1}{2}+\lambda\right)_{1+n} n !}\right. \\
= & -|\kappa|\left(\lambda+|\kappa|-\frac{1}{2}\right)^{n=|\kappa|-\lambda-\frac{1}{2}} \frac{\left(\lambda-|\kappa|+\frac{1}{2}\right)_{n}\left(\lambda+|\kappa|+\frac{1}{2}\right)_{n}}{\sum_{n=0} \sin ^{2 n+1}\left(\frac{\theta}{2}\right)}
\end{aligned}
$$

Comparison with (220) proves:

$$
V_{\mid \kappa>0}=-\frac{\lambda-|\kappa|-\frac{1}{2}}{\lambda+|\kappa|-\frac{1}{2}} V_{\mid \kappa<0} ; U V_{\mid \kappa>0}=-U V_{\mid \kappa<0}
$$

